\title{
Landau Fermi Liquid Picture of Spin Density Functional Theory: Strutinsky Approach to Quantum Dots
}

\author{
Denis Ullmo, ${ }^{1}$ Hong Jiang, ${ }^{1,2}$ Weitao Yang, ${ }^{2}$ and Harold U. Baranger ${ }^{1}$ \\ ${ }^{1}$ Department of Physics, Duke University, Durham, North Carolina 27708-0305 \\ ${ }^{2}$ Department of Chemistry, Duke University, Durham, North Carolina 27708-0354
}

(Dated: January 20, 2004)

\begin{abstract}
We analyze the ground state energy and spin of quantum dots obtained from spin density functional theory (SDFT) calculations. First, we introduce a Strutinsky-type approximation, in which quantum interference is treated as a correction to a smooth Thomas-Fermi description. For large irregular dots, we find that the secondorder Strutinsky expressions have an accuracy of about 5 percent compared to the full SDFT and capture all the qualitative features. Second, we perform a random matrix theory/random plane wave analysis of the Strutinsky SDFT expressions. The results are statistically similar to the SDFT quantum dot statistics. Finally, we note that the second-order Strutinsky approximation provides, in essence, a Landau Fermi liquid picture of spin density functional theory. For instance, the leading term in the spin channel is simply the familiar exchange constant. A direct comparison between SDFT and the perturbation theory derived "universal Hamiltonian" is thus made possible.
\end{abstract}

PACS numbers: 73.21.La, 73.23.Hk, 05.45.Mt, 71.10.Ay

\section{INTRODUCTION}

Semiconductor quantum dots are now routinely obtained using electrostatic gates or etching processes to pattern a two dimensional electron gas formed in some heterostructure (typically GaAs/AlGaAs) $\stackrel{1}{=}$ Many of their sometimes surprising properties are now reasonably well understood from a qualitative or statistical point of view $\frac{1.2}{1}$ and it is now realistic to think about using these quantum dots for some specific purpose, such as spin filtering, $\frac{3}{=}$ current or spin pumping,,$\stackrel{4}{\text { or }}$ in the context of quantum information $\frac{5}{\underline{n}}$

In this context, it becomes important to go beyond a qualitative or statistical description, and to develop tools able to predict quantitatively the properties of a specific quantum dot for given parameters. For isolated or weakly connected dots - the Coulomb Blockade regime - the Coulomb interaction between electrons plays an important role and has to be taken into account properly.

A method of choice in this regard is the density functional approach ${ }^{6.7}$ which has been widely used in theoretical modeling of quantum dots $\stackrel{\underline{8}}{=}$ More specifically, since it is necessary to describe correctly the spin degree of freedom, we have in mind a spin density functional, where each density of spin $n^{\sigma}(\mathbf{r})$, with $\sigma=\alpha, \beta$ corresponding to majority and minority spins, are treated as an independent variable. How this can be achieved in practice for a dot containing up to four hundred electrons, and for an $r_{s}$ parameter as high as four, has been demonstrated in a series of papers $, 9,10,11$

One striking feature of these calculations, however, is that the qualitative picture which emerges is somewhat unexpected in view of previous results. Indeed, within a statistical approach and assuming the classical dynamics within the nanostructure is sufficiently chaotic, one can model the wave functions in the quantum dot using Random Matrix Theory (RMT). If furthermore the Coulomb interaction is treated within the Random Phase Approximation (RPA), it is possible to derive various statistical quantities, ${ }^{12,13,14,15}$ such as the dis- tribution of spacing between Coulomb Blockade conductance peak, or the probability of occurrence of non-standard spins [that is, not zero (not one-half) for even (odd) particle number]. It turns out, for instance, that the spin density functional calculations give a larger number of "high" spins than was predicted within RMT plus RPA modeling 9 ? Such discrepancies could originate from a variety of causes, ranging from the the statistical behavior of the Kohn-Sham wave functions to an intrinsic failure of one or the other approach. The goal of this paper is to clarify this issue.

For this purpose, we need a way to understand, or to organize, the numbers obtained from the full-fledged spin density functional theory (SDFT). This can be done using the Strutinsky approximation to DFT discussed in Ref. 16, up to straightforward modifications to include the spin variable.

The Strutinsky approach is an approximation scheme, developed in the late sixties in the context of nuclear physics, in which the interference (or shell) effects are introduced perturbatively ${ }^{17.18}$ It has been since then used in many subfield of physics, $\frac{19}{19}$ including the calculation of the binding energy of metal clusters ${ }^{20}$ Standard treatments are usually limited to first-order corrections; in this case, it is similar to the Harris functiona ${ }^{121}$ familiar to the DFT literature. Here, in contrast, we use the second-order approximation ${ }^{22}$ developed in Ref. 16. We shall see that the second-order Strutinsky scheme turns out to be extremely accurate in some circumstances. Furthermore, even when it is less precise, which happens in conjunction with the occurrence of "spin contamination" in the SDFT calculations, it still provides a qualitatively correct statistical description.

The second-order Strutinsky corrections can be cast in a very natural form: ${ }^{16}$ in fact, they amount to taking into account the residual (screened) interactions between quasiparticles in a Landau Fermi liquid picture. They are thus amenable to treatment by the same RMT approach as was used previously for RPA, providing an analogue of the "universal Hamiltonian" in the case of SDFT. Since within the Strutinsky approximation the quantum dot properties are relatively transparent, 
we will then be in position to discuss the difference between SDFT results and those obtained from RMT plus RPA.

The paper is organized as follows. In Section $\amalg$ we briefly review the Strutinsky approximation as it applies to spin density functional theory. In Section [III, we consider more specifically the local density approximation from this perspective, and in particular the screened potential that it implies. Section IV] covers in detail the specific case of a model quantum dot with quartic external confining potential. This model is used to investigate the accuracy of the Strutinsky approximation for various electron densities. In Section $\nabla$ we discuss how the Fermi liquid picture emerging from the Strutinsky approximation scheme can be used, in conjunction with a random plane wave model of the wavefunctions, to analyze the peak spacing and spin the distributions resulting from the SDFT calculation. This framework also makes it possible to discuss the mechanism of spin contamination. Finally, in the last section we come back to the original question motivating this work and make use of what we understand from the Strutinsky approximation to discuss the discrepancies between SDFT calculations and RPA plus RMT predictions.

\section{STRUTINSKY APPROXIMATION FOR SPIN DENSITY FUNCTIONAL THEORY}

In the spin density functional description of a quantum dot, one considers a functional of both spin densities $\left[n^{\alpha}(\mathbf{r}), n^{\beta}(\mathbf{r})\right]$

$$
\mathcal{F}_{\mathrm{KS}}\left[n^{\alpha}, n^{\beta}\right]=\mathcal{T}_{\mathrm{KS}}\left[n^{\alpha}, n^{\beta}\right]+\mathcal{E}_{\mathrm{tot}}\left[n^{\alpha}, n^{\beta}\right],
$$

where $\sigma=\alpha, \beta$ correspond to majority and minority spins, respectively. In this expression, the second term is an explicit functional of the densities,

$$
\begin{aligned}
& \mathcal{E}_{\text {tot }}\left[n^{\alpha}, n^{\beta}\right] \equiv \int d \mathbf{r} n(\mathbf{r}) U_{\text {ext }}(\mathbf{r}) \\
& +\int d \mathbf{r} d \mathbf{r}^{\prime} n(\mathbf{r}) v_{\mathrm{int}}\left(\mathbf{r}-\mathbf{r}^{\prime}\right) n\left(\mathbf{r}^{\prime}\right)+\mathcal{E}_{\mathrm{xc}}\left[n^{\alpha}, n^{\beta}\right],
\end{aligned}
$$

where $n(\mathbf{r})=n^{\alpha}(\mathbf{r})+n^{\beta}(\mathbf{r}), U_{\text {ext }}(\mathbf{r})$ is the exterior confining potential, and the precise form of the exchange correlation term $\mathcal{E}_{\mathrm{xc}}\left[n^{\alpha}, n^{\beta}\right]$ is to be discussed in more detail in Section III $v_{\text {int }}\left(\mathbf{r}, \mathbf{r}^{\prime}\right)$ is the electron-electron interaction kernel. The presence of metallic gates can be taken into account by including an image term in addition to the bare Coulomb interaction,

$$
v_{\text {int }}\left(\mathbf{r}-\mathbf{r}^{\prime}\right)=\frac{e^{2}}{\left|\mathbf{r}-\mathbf{r}^{\prime}\right|}-\frac{e^{2}}{\sqrt{\left|\mathbf{r}-\mathbf{r}^{\prime}\right|^{2}+4 z_{d}^{2}}},
$$

where $z_{d}$ is the distance between the top confining gate and two-dimensional electron gas. The image term in the interaction kernel greatly reduces classical Coulomb repulsion between electrons at a distance larger than $z_{d}$ so that the electron density far from the boundary is quite uniform. The bare Coulomb interaction can be recovered by letting $z_{d}$ go to infinity.
The kinetic energy term $\mathcal{T}_{\mathrm{KS}}\left[n^{\alpha}, n^{\beta}\right]$, on the other hand, is expressed in terms of the auxiliary set of orthonormal functions $\psi_{i}^{(\alpha, \beta)}\left(i=1, \cdots, N_{\alpha, \beta}\right)$ such that $n^{\sigma}(\mathbf{r}) \equiv$ $\sum_{i=1}^{N_{\sigma}}\left|\psi_{i}^{\sigma}(\mathbf{r})\right|^{2}$ as

$$
\mathcal{T}_{\mathrm{KS}}\left[n^{\alpha}, n^{\beta}\right]=\frac{\hbar^{2}}{2 m} \int \sum_{\sigma=\alpha, \beta} \sum_{i=1}^{N_{\sigma}}\left|\nabla \psi_{i}^{\sigma}(\mathbf{r})\right|^{2} d \mathbf{r} .
$$

¿From the density functional Eq. (1), the ground state energy of the quantum dot containing $\left(N_{\alpha}, N_{\beta}\right)$ particles of spin $(\alpha, \beta)$ is obtained as

$$
E_{\mathrm{KS}}\left(N_{\alpha}, N_{\beta}\right)=\mathcal{F}_{\mathrm{KS}}\left[n_{\mathrm{KS}}^{\alpha}, n_{\mathrm{KS}}^{\beta}\right]
$$

where the Kohn Sham densities $\left[n_{\mathrm{KS}}^{\alpha}(\mathbf{r}), n_{\mathrm{KS}}^{\beta}(\mathbf{r})\right]$ minimize $\mathcal{F}_{\mathrm{KS}}$ under the constraint given by the number of particles of each spin. This in practice is equivalent to solving the KohnSham equations

$$
\left(-\frac{\hbar^{2}}{2 m} \nabla^{2}+U_{\mathrm{KS}}^{\sigma}(\mathbf{r})\right) \psi_{i}^{\sigma}(\mathbf{r})=\epsilon_{i}^{\sigma} \psi_{i}^{\sigma}(\mathbf{r}), \quad i=1, \ldots, N_{\sigma} .
$$

with the spin dependent self-consistent potential

$$
U_{\mathrm{KS}}^{\sigma}(\mathbf{r})=\frac{\delta \mathcal{E}_{\mathrm{tot}}}{\delta n^{\sigma}(\mathbf{r})}\left[n_{\mathrm{KS}}^{\alpha}, n_{\mathrm{KS}}^{\beta}\right] .
$$

In this section, we shall give a brief description of the second-order Strutinsky approximation as it applies to spin density functional theory. Up to the introduction of the spin indices, the derivation of this approximation follows exactly the same lines as the spinless case discussed in details in Ref. 16. We shall therefore not reproduce it here, but rather try to emphasize what exactly are the assumptions made in deriving the approximation, and then merely write down the expression we shall use in the following sections.

\section{A. Generalized Thomas-Fermi approximation}

The basic idea of the Strutinsky energy correction method is to start from smooth approximations, $E_{\mathrm{TF}}$ and $n_{\mathrm{TF}}(\mathbf{r})$, to the DFT energy $E_{\mathrm{KS}}$ and electronic density $n_{\mathrm{KS}}(\mathbf{r})$. Then, fluctuating terms are added perturbatively as an expansion in the small parameter

$$
\delta n(\mathbf{r})=n_{\mathrm{KS}}(\mathbf{r})-n_{\mathrm{TF}}(\mathbf{r}) .
$$

In the original work of Strutinsky ${ }^{17.18}$ various ways of constructing the smooth approximation have been considered. The most natural choice for $n_{\mathrm{TF}}^{\sigma}(r)$ turns out to be the solution of the Thomas-Fermi equation

$$
\frac{\delta \mathcal{F}_{\mathrm{TF}}}{\delta n^{\sigma}}\left[n^{\alpha}, n^{\beta}\right]=\mu_{\mathrm{TF}}^{\sigma}
$$

coupled with $E_{\mathrm{TF}}=\mathcal{F}_{\mathrm{TF}}\left[n_{\mathrm{TF}}^{\alpha}(r), n_{\mathrm{TF}}^{\beta}(r)\right]$. The "generalized" Thomas-Fermi functional is defined as

$$
\mathcal{F}_{\mathrm{TF}}\left[n^{\alpha}, n^{\beta}\right]=\mathcal{T}_{\mathrm{TF}}\left[n^{\alpha}, n^{\beta}\right]+\mathcal{E}_{\mathrm{tot}}\left[n^{\alpha}, n^{\beta}\right],
$$


with $\mathcal{E}_{\text {tot }}\left[n^{\alpha}, n^{\beta}\right]$ given by Eq. (2). It differs from the original spin density functional only in that the quantum mechanical kinetic energy $\mathcal{T}_{\mathrm{KS}}$, Eq. (4), is replaced by an explicit functional of the density. For two dimensional systems this takes the form $\mathcal{T}_{\mathrm{TF}}\left[n^{\alpha}, n^{\beta}\right]=\mathcal{T}_{\mathrm{TF}}^{(0)}\left[n^{\alpha}, n^{\beta}\right]+\mathcal{T}_{\mathrm{TF}}^{(1)}\left[n^{\alpha}, n^{\beta}\right]$ with

$$
\begin{aligned}
& \mathcal{T}_{\mathrm{TF}}^{(0)}\left[n^{\alpha}, n^{\beta}\right]=\frac{1}{2 N(0)} \int d \mathbf{r}\left[n^{\alpha}(\mathbf{r})^{2}+n^{\beta}(\mathbf{r})^{2}\right] \\
& \mathcal{T}_{\mathrm{TF}}^{(1)}\left[n^{\alpha}, n^{\beta}\right]=\frac{\eta}{8 \pi N(0)} \int d \mathbf{r}\left[\frac{\left(\nabla n^{\alpha}\right)^{2}}{n^{\alpha}}+\frac{\left(\nabla n^{\beta}\right)^{2}}{n^{\beta}}\right](12)
\end{aligned}
$$

where $N(0)=m / \pi \hbar^{2}$ the density of states for 2-dimensional systems and $\eta$ a dimensionless constant taken here to be 0.25 . Such a choice for the kinetic energy functional correctly takes into account the Pauli exclusion principle, and thus that an increase in kinetic energy is required to put many particle at the same space location, but fails to include fluctuations associated with quantum interference.

To illustrate this, let us for a short while the assume $\eta=$ 0 , i.e. only the first term $\mathcal{T}_{\mathrm{TF}}^{(0)}$ of the Thomas-Fermi kinetic energy is taken into account. Then, one can show that the Thomas-Fermi density fulfills the self-consistent equation

$$
n_{\mathrm{TF}}^{\sigma}(\mathbf{r})=\bar{n}^{\sigma}\left[U_{\mathrm{TF}}^{\sigma}\right](\mathbf{r})
$$

where the Thomas-Fermi potential is defined, as in Eq. (7), by

$$
U_{\mathrm{TF}}^{\sigma}(\mathbf{r})=\frac{\delta \mathcal{E}_{\mathrm{tot}}}{\delta n^{\sigma}(\mathbf{r})}\left[n_{\mathrm{TF}}^{\alpha}, n_{\mathrm{TF}}^{\beta}\right]
$$

and

$$
\bar{n}^{\sigma}\left[U_{\mathrm{TF}}^{\sigma}\right](\mathbf{r})=\int \frac{d \mathbf{p}}{(2 \pi \hbar)^{d}} \Theta\left[\mu_{\mathrm{TF}}^{\sigma}-\frac{\mathbf{p}^{2}}{2 m}-U_{\mathrm{TF}}^{\sigma}(\mathbf{r})\right]
$$

is the Weyl part of the density of states of a system of independent particles evolving under the potential $U_{\mathrm{TF}}^{\sigma}(\mathbf{r})(\Theta$ is the Heaviside step function, $d=2$ is the dimensionality, and the chemical potentials $\mu_{\mathrm{TF}}^{\sigma}$ are chosen such as to fulfill the constraints on the total number of particles with spin $\alpha$ and $\beta$ ). From its definition, $\bar{n}^{\sigma}\left[U_{\mathrm{TF}}^{\sigma}\right](\mathbf{r})$ (and as a consequence $\left.n_{\mathrm{TF}}^{\sigma}(\mathbf{r})\right)$ is a smooth function, in the sense that it can change appreciably only on the scale on which $U_{\text {ext }}(\mathbf{r})$ varies, but cannot account for the quantum fluctuations of the density on the scale of the Fermi wavelength.

The Weyl approximation is, however, only the leading term in a semiclassical expansion of the smooth part of the density of states, and higher-order corrections in $\hbar$ can be added in a systematic way. The standard way to choose $\mathcal{T}_{\mathrm{TF}}^{(1)}{ }^{19}$ is such that Eq. (13) holds, but with an approximation to the smooth density of states $\bar{n}^{\sigma}\left[U_{\mathrm{TF}}^{\sigma}\right](\mathbf{r})$ which includes both the Weyl term Eq. (15) and the first $\hbar$ corrections. For two dimensional systems, however, the corrective term computed from this prescription turns out to be zero, when the presence of $\mathcal{T}_{\mathrm{TF}}^{(1)}$ is actually useful in smoothing the Thomas-Fermi density near the boundaries of the classically allowed region. We have therefore used the phenomenological Weisäcker-like term ${ }^{19}$ Eq. (12) which plays a similar role.

\section{B. Strutinsky correction terms}

The practical implementation for the Strutinsky scheme can be summarized as follows. The first step consists in solving the generalized Thomas-Fermi equation Eq. 9, which defines a zeroth-order approximation for the ground state energy, $E_{\mathrm{TF}}$, as well as an approximation to the density of particles $n_{\mathrm{TF}}^{\sigma}(\mathbf{r})$. From this latter quantity, one derives for each spin $\sigma=\alpha, \beta$ the effective potential $U_{\mathrm{TF}}^{\sigma}(\mathbf{r})$ through Eq. 14.

The second step consists in solving the Schrödinger equations (again for each spin)

$$
\left(-\frac{\hbar^{2}}{2 m} \nabla^{2}+U_{\mathrm{TF}}^{\sigma}(\mathbf{r})\right) \phi_{i}^{\sigma}(\mathbf{r})=\tilde{\epsilon}_{i}^{\sigma} \phi_{i}^{\sigma}(\mathbf{r}), \quad i=1, \ldots, N_{\sigma} .
$$

Therefore, while the Kohn-Sham equations are both quantum mechanical in nature and self-consistent, here all the selfconsistency is left at the "classical-like" level of the ThomasFermi equation, and the quantum mechanical wave interference aspect is taken into account without self-consistency. One obtains in this way a new density

$$
\tilde{n}^{\sigma}(\mathbf{r})=\sum_{1}^{N_{\sigma}}\left|\phi_{i}^{\sigma}\right|^{2}(\mathbf{r}) .
$$

It can be seen ${ }^{16}$ that $n_{\mathrm{TF}}^{\sigma}(\mathbf{r})$ is by construction a smooth approximation to $\tilde{n}^{\sigma}(\mathbf{r})$ [this is basically the content of Eq. (13)]. Therefore

$$
\tilde{n}_{\mathrm{osc}}^{\sigma}(\mathbf{r}) \equiv \tilde{n}^{\sigma}(\mathbf{r})-n_{\mathrm{TF}}^{\sigma}(\mathbf{r})
$$

can be considered as the oscillating part of $\tilde{n}^{\sigma}$, and will describe the short scale variations of the density associated with quantum interference.

Once the eigenfunctions and eigenvalues of Eq. (16) are known, corrections to the Thomas-Fermi energy can be added perturbatively

$$
E_{\mathrm{KS}} \simeq E_{\mathrm{TF}}+\Delta E^{(1)}+\Delta E^{(2)} .
$$

The first-order correction turns out to be simply the oscillating part of the one particle energy ${ }^{16.19}$

$$
\Delta E^{(1)}=\mathcal{E}_{1 \mathrm{p}}^{\text {osc }}=\mathcal{E}_{1 \mathrm{p}}-\overline{\mathcal{E}}_{1 \mathrm{p}}
$$

with

$$
\mathcal{E}_{1 \mathrm{p}}=\sum_{i=1}^{N_{\alpha}} \tilde{\epsilon}_{i}^{\alpha}+\sum_{i=1}^{N_{\beta}} \tilde{\epsilon}_{i}^{\beta}
$$

the one particle energy, and

$$
\overline{\mathcal{E}}_{1 \mathrm{p}}=\mathcal{T}_{\mathrm{TF}}\left[n_{\mathrm{TF}}^{\alpha}, n_{\mathrm{TF}}^{\beta}\right]+\sum_{\sigma=\alpha, \beta} \int d \mathbf{r} U_{\mathrm{TF}}^{\sigma}(\mathbf{r}) n_{\mathrm{TF}}^{\sigma}(\mathbf{r})
$$

its smooth part.

The second-order correction $\Delta E^{(2)}$ can be expressed in two different ways 16

$$
\Delta E^{(2)}=\frac{1}{2} \sum_{\sigma, \sigma^{\prime}=\alpha, \beta} \int d \mathbf{r} d \mathbf{r}^{\prime} \tilde{n}_{\mathrm{osc}}^{\sigma}(\mathbf{r}) V_{\mathrm{bare}}^{\sigma, \sigma^{\prime}}\left(\mathbf{r}, \mathbf{r}^{\prime}\right) \delta n^{\sigma^{\prime}}\left(\mathbf{r}^{\prime}\right)
$$


$\Delta E^{(2) *}=\frac{1}{2} \sum_{\sigma, \sigma^{\prime}=\alpha, \beta} \int d \mathbf{r} d \mathbf{r}^{\prime} \tilde{n}_{\mathrm{osc}}^{\sigma}(\mathbf{r}) V_{\mathrm{screened}}^{\sigma, \sigma^{\prime}}\left(\mathbf{r}, \mathbf{r}^{\prime}\right) \tilde{n}_{\mathrm{osc}}^{\sigma^{\prime}}\left(\mathbf{r}^{\prime}\right)$

with

$$
V_{\text {bare }}^{\sigma, \sigma^{\prime}}\left(\mathbf{r}, \mathbf{r}^{\prime}\right)=\frac{\delta^{2} \mathcal{E}_{\mathrm{tot}}}{\delta n^{\sigma}(\mathbf{r}) \delta n^{\sigma^{\prime}}\left(\mathbf{r}^{\prime}\right)}\left[n_{\mathrm{TF}}^{\alpha}, n_{\mathrm{TF}}^{\beta}\right]
$$

$$
V_{\text {screened }}^{\sigma, \sigma^{\prime}}\left(\mathbf{r}, \mathbf{r}^{\prime}\right)=\sum_{\sigma^{\prime \prime}=\alpha, \beta} \int d \mathbf{r}^{\prime \prime}\left[\left(\frac{\delta^{2} \mathcal{T}_{\mathrm{TF}}}{\delta n^{2}}+V_{\text {bare }}\right)^{-1}\right]^{\sigma, \sigma^{\prime \prime}}\left(\mathbf{r}, \mathbf{r}^{\prime \prime}\right)\left[\frac{\delta^{2} \mathcal{T}_{\mathrm{TF}}}{\delta n^{2}} \cdot V_{\text {bare }}\right]^{\sigma^{\prime \prime}, \sigma^{\prime}}\left(\mathbf{r}^{\prime \prime}, \mathbf{r}^{\prime}\right)
$$

(The matrix inversion here should be taken with respect to both the spatial position and the spin indices.) Note that in Eq. (26) we can use

$$
\frac{\delta^{2} \mathcal{T}_{\mathrm{TF}}^{(0)}}{\delta n^{\sigma}(\mathbf{r}) \delta n^{\sigma^{\prime}}\left(\mathbf{r}^{\prime}\right)}=\frac{2}{N(0)} \delta_{\sigma, \sigma^{\prime}} \delta\left(\mathbf{r}^{\prime}-\mathbf{r}\right)
$$

and, neglecting terms involving derivatives of the ThomasFermi density,

$$
\frac{\delta^{2} \mathcal{T}_{\mathrm{TF}}^{(1)}}{\delta n^{\sigma}(\mathbf{r}) \delta n^{\sigma^{\prime}}\left(\mathbf{r}^{\prime}\right)}=-\frac{\eta}{4 \pi N(0)} \frac{\delta_{\sigma, \sigma^{\prime}}}{n_{\mathrm{TF}}^{\sigma}(\mathbf{r})} \Delta_{r^{\prime}} \delta\left(\mathbf{r}^{\prime}-\mathbf{r}\right) .
$$

The first of these second-order expressions, Eq. 23, involves the $\delta n^{\sigma}(\mathbf{r})$ which are in principle unknown. It is therefore not useful if one is trying to avoid performing the full Kohn-Sham calculation. Here, however, we shall also do this full self-consistent calculation in order to evaluate the accuracy of the Strutinsky scheme; we therefore know $\delta n^{\sigma}(\mathbf{r})$. In this case Eq. 23) turns out to be somewhat simpler to compute than Eq. (24). This is not, however, because of the matrix inversion in Eq. 26 - for the case we shall consider, the screening length is much smaller than the size of the system, allowing this inversion to be done analytically. Rather, the resulting screened potential will turn out to be diagonal neither in the position nor in the momentum representation (see next section), in contrast to $V_{\text {bare }}^{\sigma, \sigma^{\prime}}$ which is the sum of two parts, one diagonal in the position and the other in the momentum representation. Since Eq. (23) and (24) are essentially at the same level of precision, we shall in the following mainly use the first one Eq. 23); we will apply Eq. (24) only with an approximate $V_{\text {screened }}$ which is diagonal in the momentum representation, thus inducing additional errors that are not intrinsic to the Strutinsky scheme.

\section{Applicability of second-order Strutinsky expressions}

Although we shall not reproduce here the derivation of Eq. (23) (see again Ref. 16 for details), the condition of applicability of this equation can be understood easily by comparing the Kohn-Sham equations Eq. (6) with the ones defined by the Thomas-Fermi potential $U_{\mathrm{TF}}^{\sigma}(\mathbf{r})$, Eq. (16). We see there that the Kohn-Sham wave functions $\psi_{i}^{\sigma}$ and their approximations $\phi_{i}^{\sigma}$ are defined through Schrödinger equations that differ only by a difference in the potential term

$$
\begin{aligned}
\delta U^{\sigma}(\mathbf{r}) & =U_{\mathrm{KS}}^{\sigma}(\mathbf{r})-U_{\mathrm{TF}}^{\sigma}(\mathbf{r}) \\
& \simeq \sum_{\sigma^{\prime}=\alpha, \beta} \int d \mathbf{r}^{\prime} V_{\text {bare }}^{\sigma, \sigma^{\prime}}\left(\mathbf{r}, \mathbf{r}^{\prime}\right) \delta n^{\sigma^{\prime}}\left(\mathbf{r}^{\prime}\right)
\end{aligned}
$$

with $V_{\text {bare }}\left(\mathbf{r}, \mathbf{r}^{\prime}\right)$ defined by Eq. 25.

The main approximation in the derivation of Eq. (23) is that the $\delta U(\mathbf{r})$ can be taken into account by a second-order perturbative calculation. In general, this implies that the non diagonal matrix elements $(\delta U)_{i j}^{\sigma}=\left\langle\phi_{i}^{\sigma}\left|\delta U^{\sigma}\right| \phi_{j}^{\sigma}\right\rangle(i \neq j)$ should typically be smaller than the mean level spacing $\Delta$ between one particle energies. More specifically, since the only relevant errors are ones modifying the Slater determinant formed by the occupied orbitals, the actual condition is that the matrix element between the first unoccupied orbital and the last occupied orbital is smaller than the spacing between these level, that is

$$
\left\langle\phi_{N_{\sigma}+1}^{\sigma}\left|\delta U^{\sigma}\right| \phi_{N_{\sigma}}^{\sigma}\right\rangle \ll\left(\tilde{\epsilon}_{N_{\sigma}+1}-\tilde{\epsilon}_{N_{\sigma}}\right) \quad(\sigma=\alpha, \beta) .
$$

A good accuracy, on the scale of the one particle mean level spacing, of the Strutinsky approximation in the form Eq. 23. is equivalent to the above condition.

Now, Eq. (30) again involves $\delta n^{\sigma}(\mathbf{r})$, which is unknown. It is therefore not possible to prove rigorously that it should be fulfilled. It is possible, however, to show the consistency of such an assumption. If we assume that

(i) $\left|\left\langle\phi_{i}^{\sigma}\left|\delta U^{\sigma}\right| \phi_{j}^{\sigma}\right\rangle\right| \ll \Delta$ for all pairs of orbitals $(i, j)$ and $\sigma=\alpha, \beta$, so that $\delta U^{\sigma}$ can be treated perturbatively, and

(ii) the oscillating parts of the Kohn-Sham densities $\left(n_{\mathrm{KS}}\right)_{\mathrm{osc}}^{\sigma}(\mathbf{r})$ do not differ significantly from $\tilde{n}_{\mathrm{osc}}^{\sigma}(\mathbf{r})$, implying that $\delta n^{\sigma}(\mathbf{r}) \simeq \delta \bar{n}^{\sigma}(\mathbf{r})+\tilde{n}_{\mathrm{osc}}^{\sigma}(\mathbf{r})$,

then it can be shown that

(a)

$$
\delta U^{\sigma}(\mathbf{r})=\sum_{\sigma^{\prime}=\alpha, \beta} \int d \mathbf{r}^{\prime} V_{\text {screened }}\left(\mathbf{r}, \mathbf{r}^{\prime}\right) \tilde{n}_{\mathrm{osc}}\left(\mathbf{r}^{\prime}\right)
$$


which immediately yields Eq. (24) from (23), and

(b) $\left\langle\delta U_{i j}^{2}\right\rangle / \Delta^{2}$ and $\left\langle\left[\left(n_{\mathrm{KS}}\right)_{\mathrm{osc}}^{\sigma}(\mathbf{r})-\tilde{n}_{\mathrm{osc}}^{\sigma}(\mathbf{r})\right]^{2}\right\rangle /\left\langle\left[\delta n^{\sigma}(\mathbf{r})\right]^{2}\right\rangle$ are both negligible for a large dot for which the chaotic motion in the potential $U_{\mathrm{TF}}^{\sigma}$ allows modeling of the wave functions in terms of random plane waves (being of order $\ln g / g$ with $g$ the dimensionless conductance of the dot).

\section{THE SCREENED INTERACTION IN THE LOCAL DENSITY APPROXIMATION}

The second order Strutinky corrections, Eqs. 23) or (24), involve the bare and screened interactions Eqs. (25) and (26). In this section, we shall consider the particular form these interactions take for the exchange correlation term we use to perform the actual SDFT calculations, namely the local spin density approximation

$$
\mathcal{E}_{\mathrm{xc}}\left[n^{\alpha}, n^{\beta}\right] \simeq \int d \mathbf{r} n(\mathbf{r}) \epsilon_{\mathrm{xc}}(n(\mathbf{r}), \zeta(\mathbf{r})),
$$

where $\zeta(\mathbf{r})=\left(n^{\alpha}-n^{\beta}\right) / n$ is the polarization of the electron gas, and $\epsilon_{\mathrm{xc}}$ is the exchange plus correlation energy per electron for the uniform electron gas with polarization $\zeta$. We furthermore use Tanatar and Ceperley's form of $\epsilon_{\mathrm{xc}}$ at $\zeta=0$ and 1,23 and the interpolation $\epsilon_{\mathrm{xc}}(n, \zeta)=\epsilon_{\mathrm{xc}}(n, 0)+$ $f(\zeta)\left[\epsilon_{\mathrm{xc}}(n, 1)-\epsilon_{\mathrm{xc}}(n, 0)\right]$ for arbitrary polarization, with $f(\zeta)=\left[(1+\zeta)^{3 / 2}+(1-\zeta)^{3 / 2}-2\right] /\left(2^{3 / 2}-2\right)$. Recently, Attaccalite et al ${ }^{24}$ parameterized a new LSDA exchangecorrelation functional form based on more accurate quantum Monte Carlo calculations (see e.g. Ref. 25) that include spin polarization explicitly. We have, however, checked that for the quantities in which we are interested here, this new functional introduces only minor modifications; we shall therefore use Tanatar-Ceperley's form for our discussion.

From the expression of the functional $\mathcal{E}_{\mathrm{xc}}\left[n^{\alpha}, n^{\beta}\right]$, the bare and screened interaction potentials Eq. 25 and 26, needed for the evaluation of the second-order Strutinsky corrections, are easily computed. The bare interaction is the sum of two terms $V_{\text {bare }}=V_{\text {coul }}+V_{\text {xc }}$. The Coulomb interaction

$$
V_{\text {coul }}\left(\mathbf{r}, \mathbf{r}^{\prime}\right)=v_{\text {int }}\left(\left|\mathbf{r}-\mathbf{r}^{\prime}\right|\right) \cdot\left(\begin{array}{cc}
1 & 1 \\
1 & 1
\end{array}\right)
$$

is independent of both the density and spin. The matrix structure here refers to the spin indices $\alpha$ and $\beta$. The exchange correlation term is local and can be expressed as

$$
\begin{aligned}
& V_{\mathrm{xc}}\left(\mathbf{r}, \mathbf{r}^{\prime}\right)=-2 \pi a_{0} e^{2} \delta\left(\mathbf{r}-\mathbf{r}^{\prime}\right) \\
& \times\left(\begin{array}{cc}
v_{a}(n(\mathbf{r}), \zeta(\mathbf{r})) & v_{b}((n(\mathbf{r}), \zeta(\mathbf{r})) \\
v_{b}(n(\mathbf{r}), \zeta(\mathbf{r})) & v_{a}(n(\mathbf{r}),-\zeta(\mathbf{r}))
\end{array}\right),
\end{aligned}
$$

where $v_{a}$ and $v_{b}$ are obtained from the partial derivative of $\epsilon_{\mathrm{xc}}(n, \zeta)$ [defined in Eq. (32)]. In all numerical calculations we shall keep entirely the dependence of $v_{a}$ and $v_{b}$ on the polarization $\zeta$. However, this latter will usually be relatively small, and $v_{a}$ and $v_{b}$ contains already second derivatives of the functional $\mathcal{E}_{\mathrm{xc}}$ with respect to the polarization.

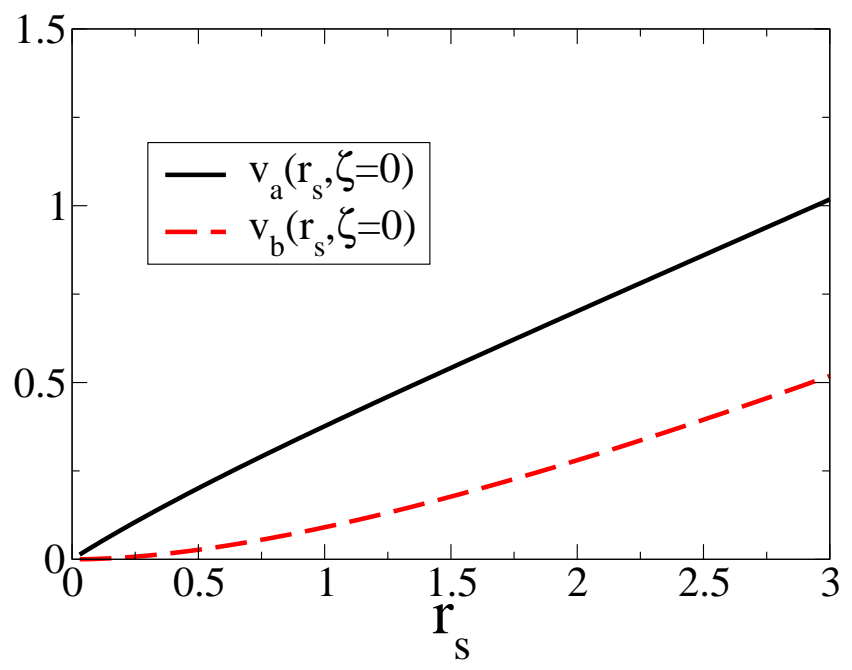

FIG. 1: (Color online) The functions $v_{a}(n, 0)$ and $v_{b}(n, 0)$, which define the functional second derivative of $\mathcal{E}_{\mathrm{xc}}\left[n^{\alpha}, n^{\beta}\right]$ [see Eq. [34], as a function of the parameter $r_{s}=1 / \sqrt{\pi a_{0}^{2} n}$.

We shall therefore proceed assuming $v_{a}(n, \zeta) \simeq v_{a}(n, 0)$ and $v_{b}(n, \zeta) \simeq v_{b}(n, 0)$. The dependence of these functions on the parameter $r_{s}=\left(\pi a_{0}^{2} n\right)^{-1 / 2}$, with $a_{0}=\hbar^{2} / m e^{2}$ the 2D Bohr radius, is shown in Fig 1

Turning now to the screened interaction $V_{\text {screened }}\left(\mathbf{r}, \mathbf{r}^{\prime}\right)$, it is useful to switch to the variables $(\mathbf{R}, \mathbf{l})=\left(\left(\mathbf{r}^{\prime}+\mathbf{r}\right) / 2,(\mathbf{r}-\right.$ $\left.\left.\mathbf{r}^{\prime}\right)\right)$. The Fourier transform of $V_{\text {bare }}(\mathbf{R}, \mathbf{l})$ with respect to $\mathbf{l}$ reads

$$
\begin{aligned}
\hat{V}_{\text {bare }}(\mathbf{R}, \mathbf{q})= & 2 \pi e^{2} a_{0} v_{c}(\mathbf{q}) \cdot\left(\begin{array}{ll}
1 & 1 \\
1 & 1
\end{array}\right) \\
& -2 \pi a_{0} e^{2}\left(\begin{array}{cc}
v_{a}(\mathbf{R}) & v_{b}(\mathbf{R}) \\
v_{b}(\mathbf{R}) & v_{a}(\mathbf{R})
\end{array}\right)
\end{aligned}
$$

where

$$
v_{c}(\mathbf{q}) \equiv \frac{1}{a_{0}|\mathbf{q}|}\left(1-e^{-2 z_{d} q}\right)
$$

(again, the pure Coulomb case is recovered by letting the distance to the top gate $z_{d}$ go to infinity). This can by further simplified by diagonalizing the matrix in the spin indices: the eigenvectors are the "charge channel" $\vec{c}=(\vec{\alpha}+\vec{\beta}) / \sqrt{2}$ and the "spin channel" $\vec{s}=(\vec{\alpha}-\vec{\beta}) / \sqrt{2}$. The eigenvalues are

$$
\lambda_{c}^{\text {bare }}(\mathbf{R}, \mathbf{q})=\frac{2}{N(0)}\left\{2 v_{c}(q)-\left[v_{a}(\mathbf{R})+v_{b}(\mathbf{R})\right]\right\}
$$

in the charge channel and

$$
\lambda_{s}^{\text {bare }}(\mathbf{R}, \mathbf{q})=\frac{2}{N(0)}\left[v_{b}(\mathbf{R})-v_{a}(\mathbf{R})\right]
$$

in the spin channel [note $2 \pi a_{0} e^{2}=2 / N(0)$ ].

Because the screening length is short on the scale for which the smooth part of the electronic density varies appreciably, the operator $\delta^{2} \mathcal{T}_{\mathrm{TF}} / \delta n^{2}+V_{\text {bare }}$ can be inverted by treating 
the variable $\mathbf{R}$ as a parameter, appearing thus diagonal in the $\mathbf{q}$ representation. One obtains in this way

$$
\hat{V}_{\text {screened }}(\mathbf{R}, \mathbf{q})=\left(\begin{array}{cc}
\left(\lambda_{c}+\lambda_{s}\right) / 2 & \left(\lambda_{c}-\lambda_{s}\right) / 2 \\
\left(\lambda_{c}-\lambda_{s}\right) / 2 & \left(\lambda_{c}+\lambda_{s}\right) / 2
\end{array}\right),
$$

in terms of the eigenvalues

$$
\lambda_{c}(\mathbf{R}, \mathbf{q})=\frac{2}{N(0)} \frac{2 v_{c}(q)-\left[v_{a}(\mathbf{R})+v_{b}(\mathbf{R})\right]}{1+g(q)\left[2 v_{c}(q)-\left(v_{a}(\mathbf{R})+v_{b}(\mathbf{R})\right)\right]}
$$

for the charge channel and

$$
\lambda_{s}(\mathbf{R}, \mathbf{q})=\frac{2}{N(0)} \frac{\left[v_{b}(\mathbf{R})-v_{a}(\mathbf{R})\right]}{1+g(q)\left[v_{b}(\mathbf{R})-v_{a}(\mathbf{R})\right]}
$$

for the spin channel. The $\mathbf{R}$ dependence of $\lambda_{c}$ and $\lambda_{s}$ arises from $v_{a}(n(\mathbf{R}))$ and $v_{b}(n(\mathbf{R}))$, and therefore from the local value of the density (that is, of the parameter $r_{s}$ ) at the location the interaction is taking place. Furthermore, the function

$$
g(q)=\left(1+\eta \frac{q^{2}}{8 \pi n_{\mathrm{TF}}(\mathbf{R})}\right)^{-1}
$$

would just be 1 in the absence of the $\hbar$ correction $\mathcal{T}_{\mathrm{TF}}^{(1)}$ to the Thomas-Fermi kinetic energy term; it prevents effective screening to take place for large momenta.

\section{THE GATED QUARTIC OSCILLATOR MODEL}

To evaluate the accuracy of the Strutinsky approximation scheme, we consider a two dimensional model system for which the electrons are confined by a quartic potential

$$
U_{\mathrm{ext}}(x, y)=a\left[\frac{x^{4}}{b}+b y^{4}-2 \lambda x^{2} y^{2}+\gamma\left(x^{2} y-y^{2} x\right) r\right] .
$$

The role of the various parameters of $U_{\text {ext }}(x, y)$ is the following: $a$ controls the total electronic density (i.e. the parameter $r_{s}$ ), and therefore the relative strength of the Coulomb interaction; $\lambda$ allows one to place the system in a regime where the corresponding classical motion is essentially chaotic; finally, $b$ and $\gamma$ have been introduced to lower the symmetry of the system. In the following sections, we use [a1] to denote the parameter value $a=10^{-1} \times E_{R} / a_{0}^{4}$ (with $E_{R}=e^{2} / 2 a_{0}$ ), and [a4] for $a=10^{-4} \times E_{R} / a_{0}^{4}$, which at $N=120-200$ correspond to $r_{s} \simeq 0.3$ and 1.3 , respectively. We use $\lambda=0.53$ and $\gamma=0.2$ in our calculations unless specified otherwise. In addition to this 2-dimensional potential, we assume the existence of a metallic gate some distance $z_{d}$ away from the 2D electron gas whose purpose is to cut off the long-range part of the Coulomb interaction. This gate is placed sufficiently far from the electrons to prevent the formation of a density deficit in the center of the potential well without modifying qualitatively the quantum fluctuation. In practice, we take $z_{d}$ about $0.75 a_{0}$ for [a1] , and $2.5 a_{0}$ for [a 4 ] ${ }^{32}$

\section{A. Electronic densities}

For any set of parameters defining the potential Eq. 43, and for any number of up and down electrons $\left(N^{\alpha}, N^{\beta}\right)$, we can compute the Kohn-Sham energies $E_{\mathrm{KS}}\left[N^{\alpha}, N^{\beta}\right]$ and densities $n_{\mathrm{KC}}^{\sigma}(\mathbf{r})$ following the approach described in detail in Ref. 10. For the Strutinsky approximation, the only part which presents some degree of difficulty is actually the Thomas-Fermi calculation, for which we have developed a new conjugate-gradient method which turns out to be extremely efficient. ${ }^{26}$ Once $n_{\mathrm{TF}}^{(\alpha, \beta)}(\mathbf{r})$ are known, the effective potentials $U_{\mathrm{TF}}^{(\alpha, \beta)}(\mathbf{r})$ and the corresponding densities $\tilde{n}^{(\alpha, \beta)}(\mathbf{r})$ follow immediately.

Figure 2$]$ shows the densities $n_{\mathrm{TF}}(\mathbf{r}), n_{\mathrm{KS}}(\mathbf{r}), \delta n(\mathbf{r})$, and $\tilde{n}_{\text {osc }}(\mathbf{r})$ for the ground state with $N=200$ electrons $\left(N_{\alpha}=\right.$ $\left.N_{\beta}=100\right)$ of the gated quartic oscillator system with parameter [a4], corresponding to an interaction parameter of $r_{s}=1.3$. Noting in particular the difference of scale between the upper and lower panels, one can observe that the ThomasFermi density already is a very good approximation to the exact one, and that $\delta n(\mathbf{r})$ is a small oscillating correction, of the same magnitude as the oscillating part of $\tilde{n}(\mathbf{r})$. Apparent also on this figure is the fact that the largest errors are located at the boundary of the dot where corrections to the Weyl density of higher order in $\hbar$ are the largest. To make this more visible, we plot in Fig. 3 the densities $\delta n(\mathbf{r})$ and $\tilde{n}_{\mathrm{osc}}(\mathbf{r})$ along a cut on a diagonal of the confining potential for two sets of parameters. This makes it clear that $\tilde{n}_{\text {osc }}(\mathbf{r})$ is an oscillating function only in the interior of the dot, but has a proportionally large secular component at the boundary. As a consequence, choosing correctly the term $\mathcal{T}_{\mathrm{TF}}^{(1)}$ of the Thomas-Fermi kinetic energy term is actually important to obtain good accuracy. We have therefore determined the parameter $\eta=0.25$ of this functional by imposing that $\mathcal{E}_{1 \mathrm{p}}^{\mathrm{osc}}[N]$ oscillates around zero, rather than having a significant mean value. As an illustration, we also plot in Fig. 3 the same quantities but for a calculation where a value $\eta=1$ has been used for the Thomas-Fermi kinetic energy correcting term. We see that this increases significantly the error in the Thomas-Fermi density at the boundary, reducing the effectiveness of the Strutinsky approximation.

\section{B. Ground state energies}

With the expressions Eqs. (35) and 39) of the bare and screened interactions, and using the known eigenvalues and eigenfunctions for the Schrödinger equations Eqs. (6) and (16), the Strutinsky approximation for the total energy, including the order one Eq. (20) and order two Eqs. (23) or 24) corrections, can be computed for any $\left(N_{\alpha}, N_{\beta}\right)$. As for the full spin density calculations, the ground state energy for a given total number of electron $N$ is then obtain as the minimum over $\left(N_{\alpha}, N_{\beta}\right)$ with $N_{\alpha}+N_{\beta}=N$ of these energies.

Let us now consider these ground state energies for a choice of parameters [a1], such that the coefficient $r_{s}=0.3$ is still smaller than one and thus the Coulomb interaction is not very large compared to the kinetic energy of the particles. 


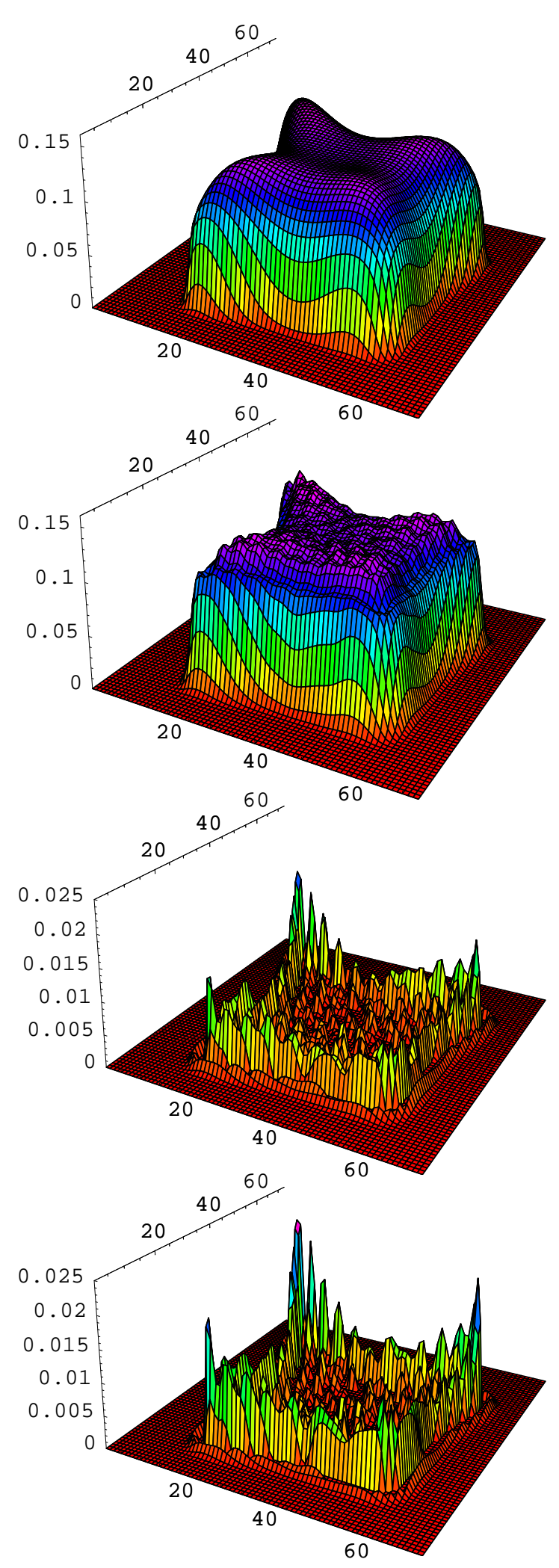

FIG. 2: (Color online) Particle density for the parameters [a4] for a system of $N=200$ electrons $\left(N_{\alpha}=N_{\beta}=100\right)$. From top to bottom: $n_{\mathrm{TF}}(\mathbf{r}) ; n_{\mathrm{KS}}(\mathbf{r}) ; \delta n(\mathbf{r})$; and $\tilde{n}_{\mathrm{osc}}(\mathbf{r})$. Note that $n_{\mathrm{TF}}$ is a smooth approximation to $n_{\mathrm{KS}}$ and that $\delta n(\mathbf{r})$ and $\tilde{n}_{\mathrm{osc}}(\mathbf{r})$ are very similar.
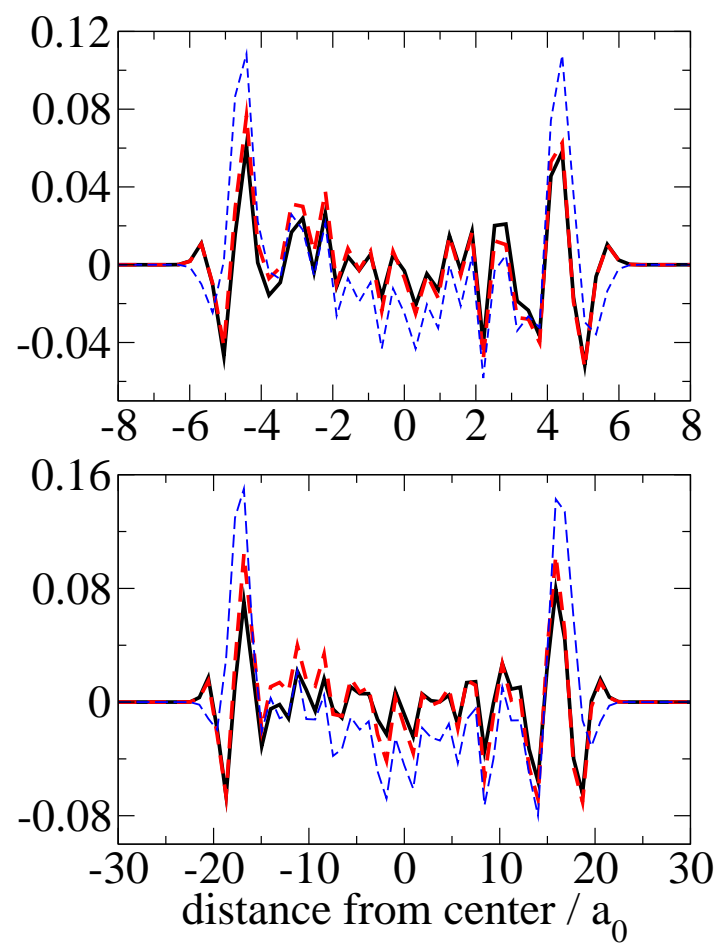

FIG. 3: (Color online) Diagonal cut of particle densities $\delta n(\mathbf{r})$ (solid) and $\tilde{n}_{\text {osc }}(\mathbf{r})$ (dashed), in units of the average Thomas-Fermi density $n_{\mathrm{TF}}$ inside the dot. Upper panel: parameters [a1] $\left(r_{s} \simeq 0.3\right)$; Lower panel parameters [a4] $\left(r_{s} \simeq 1.3\right)$. The dot contains $N=200$ electrons $\left(N_{\alpha}=N_{\beta}=100\right)$. The thin dashed lines correspond to $\tilde{n}_{\mathrm{osc}}(\mathbf{r})$ from a less accurate choice of the Thomas-Fermi kinetic energy term, namely $\eta=1.0$ instead of 0.25 .

Fig. 4 displays the corresponding difference between Strutinsky and Kohn-Sham ground-state energies, in mean level spacing units, for a number of electrons ranging from 50 to 200 . For the upper panel, $E_{\mathrm{ST}}[N]$ is obtained from the expression Eq. (23) using the bare interaction and requiring the knowledge of the exact $\delta n^{(\alpha, \beta)}$. For the lower panel, $E_{\mathrm{ST}^{*}}[N]$ is obtained from Eq. 23], which involves the approximate screened interaction, but only the knowledge of $\tilde{n}_{\mathrm{osc}}^{(\alpha, \beta)}(\mathbf{r})$.

The first observation that can be made on that figure is that the second form of the Strutinsky approximation appears substantially less accurate than the first one. As mentioned earlier, this is, however, probably due to the fact that, because our code was devised to handle only two body interactions that were diagonal either in position or in momentum representation, we had to use in that calculation an approximation where, for the screened interaction, the local value of the parameter $r_{s}(\mathbf{r})=\sqrt{1 / \pi n_{\mathrm{TF}}(\mathbf{r})}$ has been replaced by its mean value .

Indeed, a second feature immediately visible on Fig. 4 is the presence of a net trend in the energy differences between Kohn-Sham and Strutinsky calculations. This secular term is related to the non-oscillating component of $\tilde{n}_{\mathrm{osc}}(\mathbf{r})$ and $\delta n(\mathbf{r})$ visible on the lower panels of Figs. 2 and 3 at the boundary of the quantum dots. This can be checked by using a less accurate Thomas-Fermi approximation (e.g. with $\eta=1$ for the 


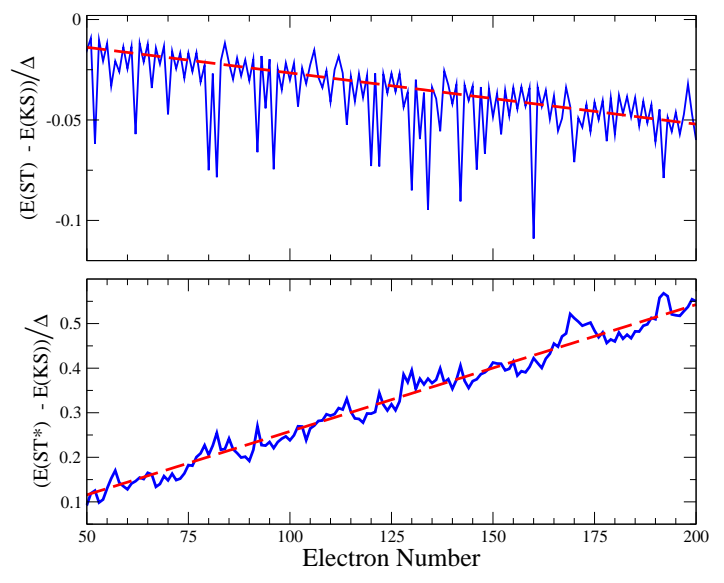

FIG. 4: (Color online) Difference between Strutinsky and KohnSham ground state energies, in units of the mean level spacing, as a function of the number of electrons, for the high density dot [a1] $\left(r_{s} \simeq 0.3\right)$. Upper panel: $E_{\mathrm{ST}}[N]$ obtained from Eq. [23); Lower panel: $E_{\mathrm{ST}^{*}}[N]$ obtained from Eq. 24]. In both cases, the difference shows small fluctuations (a few percent) about a linear secular trend (dashed line is fit).

correcting term of the kinetic energy $\mathcal{T}_{\mathrm{TF}}^{(1)}$ ), and noticing that this secular term in the deviation increases noticeably while the fluctuations remain less affected. Since the total density of electrons at the boundary of the dot is significantly lower than its average value, it is relatively natural that in our approach, this secular deviation is made significantly worse in the second form of our approximation.

The secular deviation is not completely negligible in terms of the mean level spacing. The relevant scale for the smooth part of the ground energies is, however, the charging energy, compared to which these secular corrections are extremely small. To focus on the fluctuating part, we therefore remove the secular term (i.e. the straight lines in Fig. 4), obtaining in this way Fig. 5

Let us consider first the upper panel, where the first form of the Strutinsky approximation has been used. We observe that the fluctuating part of the error is usually extremely small, typically of order a percent of a mean level spacing, and that this is mainly an oscillation between odd and even number of particles in the system. Nevertheless, in a few circumstances significantly larger deviations are observed, with a magnitude typically five percent of a mean level spacing and a sign which is always negative.

To understand the origin of these larger deviations, it is useful to correlate them with the occurrence of spin contamination, that is to situations where the SDFT calculations break the spin rotation symmetry. Since the actual spin is a somewhat ill-defined quantity in a spin density calculation, we need however first to specify what we understand by this. Indeed, in spin density functional theory the difference $N_{\alpha}-N_{\beta}$ can be interpreted as twice the component $S_{z}$ of the quantum dot total spin. Another quantity that can be easily computed is the mean value $S(S+1)$ of the operator $\vec{S}^{2}$ for the Slater determinant formed by the Kohn-Sham orbitals $\psi_{i}^{\sigma}, i=1, \cdots, N_{\sigma}$, which can be expressed as $S(S+1)=S_{z}\left(S_{z}+1\right)+\delta S$ with

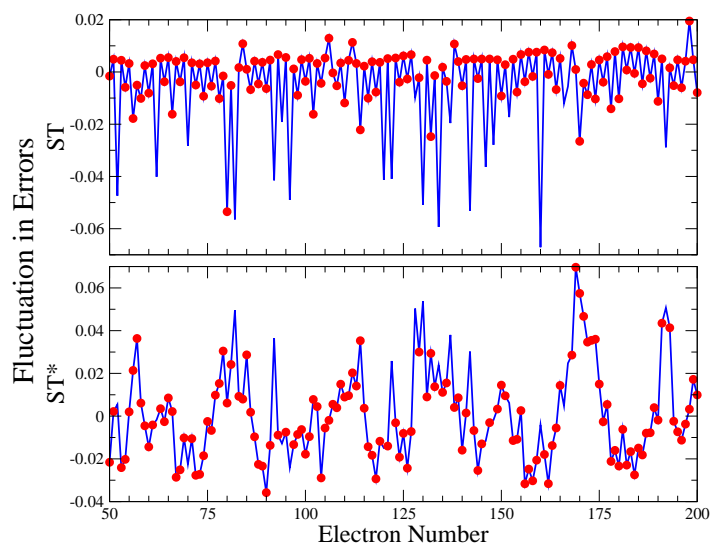

FIG. 5: (Color online) Difference between Strutinsky and KohnSham ground state energies as in Fig. 4 but now with the secular trend removed. Solid: all ground states; Dots: ground states without significant spin contamination. Note the excellent agreement in the case of the first Strutinsky form $E_{\mathrm{ST}}$ (upper panel) - of order 1 percent - when spin contamination is not present. In the case of the second form $E_{\mathrm{ST}^{*}}$, the agreement is still very good (lower panel).

the "spin contamination" $\delta S$ given by 27

$$
\delta S=N_{\beta}-\sum_{i, j \leq N_{\beta}}\left|\left\langle\psi_{i}^{\alpha} \mid \psi_{j}^{\beta}\right\rangle\right|^{2} .
$$

From this expression, one sees that if all occupied $\beta$-orbitals are identical to the corresponding $\alpha$-orbitals, $\delta S=0$, and it is presumably reasonable to interpret $\left(N_{\alpha}-N_{\beta}\right) / 2$ as the system's total spin. However, as soon as different-spin orbitals start to differ, $\delta S$ can take any positive value smaller than $N_{\beta}$, signaling that, at least, there is some ambiguity in the assessment of the total spin of the system. In Fig. 6 we have plotted the ground state spin contamination $\delta S[N]$ as a function of the particle number. For [a1], the spin contamination is usually negligible, except in some few cases where $\delta S$ 's of order one half or so are encountered.

Coming back to the Strutinsky approximation, we can use the information from Fig. 6 to exclude in Fig. [5 the ground states with significant spin contamination. The remaining points correspond to the dotted symbols in this figure. In the upper panel, we see that there is almost a one to one correspondence between larger errors and spin contamination.

Turning to the lower panel in Fig. 5 we see that the further approximations in treating the screening used in evaluating Eq. (23) do degrade the accuracy of the ground state energy somewhat. Still, ST* gives the fluctuating part of the energy to within a few percent. For the spin contamination, no particular correlation is seen, presumably again because the overall agreement is slightly spoiled by the approximation we made for the screened Coulomb interaction.

In lower density (larger $r_{s}$ ) more realistic dots modeled by the parameter set [a4], spin contamination in KS ground states is much more pronounced, as shown in the lower panel of Fig. 6- it is, in fact, always significant. In conjunction, Fig. [7 shows that the accuracy of both ST [i.e. using Eq. (20)] and $\mathrm{ST}^{*}$ [i.e. using Eq. (23)] becomes worse. As at higher density, 


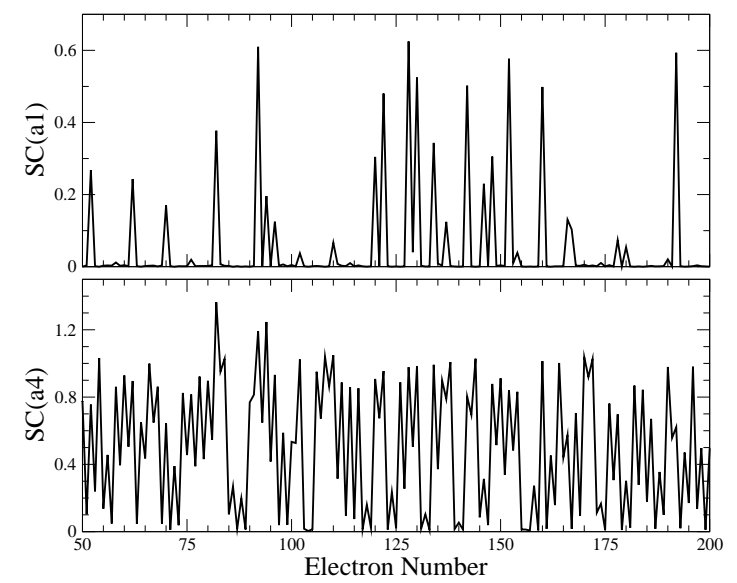

FIG. 6: Spin contamination $\delta S[N]$ as a function of the number of particles. The spin contamination is infrequent at high density ([a1], upper panel), but becomes frequent and substantial for $r_{s} \simeq 1.3$ ([a4], lower panel).

the main error is a secular trend: in the case of ST* it attains a value of several mean level spacings, due presumably to the approximations made in treating the screening. After removing the secular deviation, however, the fluctuation in the errors of ST and ST* ground state energy is still quite small: the rms is $0.05 \Delta$ in ST and $0.06 \Delta$ in ST*. Thus for characterizing the fluctuating part of the ground state energy, ST* is nearly as good as ST, a property we expect to remain valid at larger $r_{s}$.

\section{Coulomb Blockade peak spacings and spin distribution}

In the previous subsection, we have considered the accuracy of the Strutinsky approximation for individual ground state energies. We found that as long as no significant amount of spin contamination is present in the SDFT calculation, the Strutinsky result provides an excellent approximation when Eq. 23. is used, and a good, though slightly degraded one, with Eq. (24). In this latter case, it is probable that the additional errors come mainly from the neglect of the local density dependence in the screened interaction rather than to the Strutinsky approximation itself. We shall come back to the issue of spin contamination in the next section, and sketch an extension of the theory that would make it suitable to deal with the spin contaminated case.

Before doing so, however, we shall address another question, namely how well, even in the case where a one-to-one comparison of ground state energies can imply an error of a fraction of mean level spacing, are the statistical properties of the quantum dots described within the Strutinsky approximation. For instance, we have in mind the distribution of ground state spin $S_{z}[N]$ or of ground state energy second difference $s[N]=E_{\mathrm{KS}}[N+1]+E_{\mathrm{KS}}[N-1]-2 E_{\mathrm{KS}}[N+1]$. This latter quantity is accessible experimentally by measuring the spacing between conductance peaks in the Coulomb Blockade regime, and will be referred to below as the "peak spacing".

In Fig. 8, both peak spacing and spin distributions are plot-

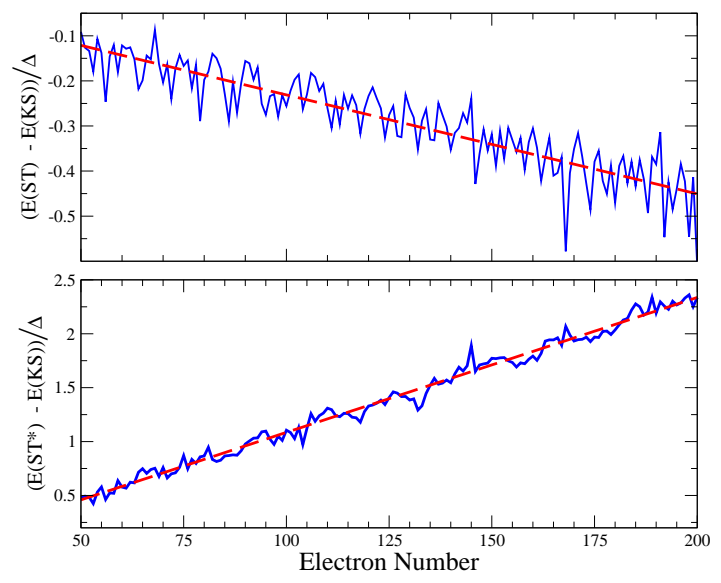

FIG. 7: (Color online) Difference between Strutinsky and KohnSham ground state energies, in units of the mean level spacing, as a function of the number of electrons, for the $r_{s} \simeq 1.3$ dot [a4]. Upper panel: $E_{\mathrm{ST}}[N]$; Lower panel: $E_{\mathrm{ST}^{*}}[N]$. The linear secular trend (dashed line is a fit) is now significantly larger than for [a1] (compare with Fig. 4), but the fluctuation about this trend remains small (of order 5 percent).

ted for two interaction strength regime ([a1] and [a4]) using either Kohn-Sham results or one or the other form of the Strutinsky approximation. In the small $r_{s}$ case, the agreement is naturally excellent, but we see that even for the higher $r_{s}$ case, both forms of the Strutinsky approximation give a fairly good approximation - certainly they provide a qualitatively correct description.

\section{FERMI LIQUID PICTURE}

It is not possible to develop a real Landau Fermi liquid theory for quantum dots because the mesoscopic fluctuations prevent any Taylor expansion of the free energy in terms of occupation number. (Landau theory basically assumes that the excitation energies are the smallest energy scales of the problem, which is clearly not true for mesoscopic systems because of variation on the scale of the mean level spacing.) However, discussing what we may call a Landau Fermi liquid "picture", in the sense that the low energy physics is described by a renormalized weak interaction, is still something meaningful.

In that sense, what Eqs. (19)-26 express is that SDFT, in the limit where the Strutinsky approximation scheme is accurate, is equivalent to a Landau Fermi liquid picture, where quasiparticles with spin $\sigma=\alpha, \beta$ evolve in the effective potential $U_{\mathrm{TF}}^{\sigma}$ and interact through a residual weak interaction $V_{\text {screened }}^{\sigma, \sigma^{\prime}}\left(\mathbf{r}, \mathbf{r}^{\prime}\right)$ that can be taken into account as a perturbation. The only unusual feature is the absence of an exchangelike contribution to the total energy. Indeed the main role of the exchange correlation functional $\mathcal{E}_{\mathrm{xc}}\left[n_{\alpha}, n_{\beta}\right]$ is to make the interaction between same spin particles different from the one between opposite spins.

Since moreover we have chosen the confining potential in such a way that the classical motion within our model 

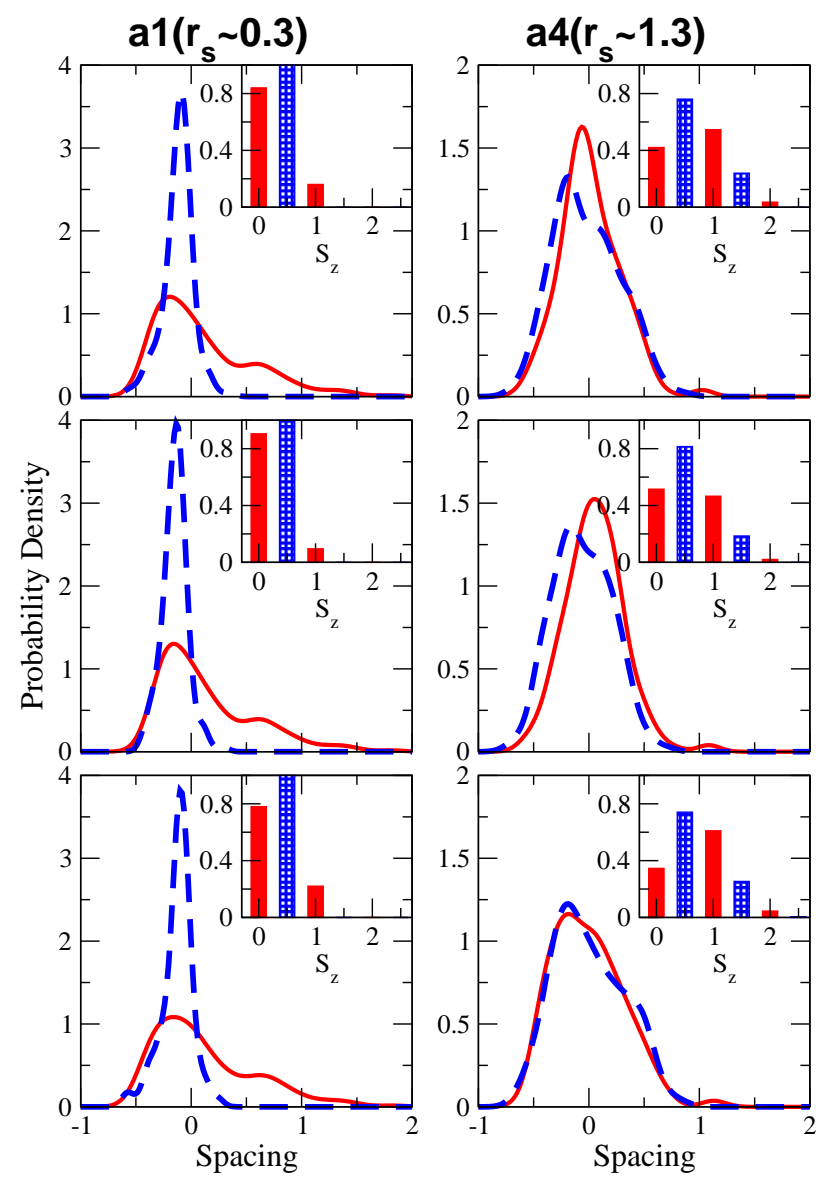

FIG. 8: (Color online) Spin and peak spacing distributions for the cases [a1] (left column) and [a4] (right column). Solid: even $N$; Dashed: odd $N$. The statistics are obtained for $N=120-200$ with $(\lambda, \gamma)=(0.53,0.2),(0.565,0.2),(0.6,0.1),(0.635,0.15)$ and $(0.67,0.1)$. From top to bottom: Kohn-Sham, first, and second form of the Strutinsky approximation. Agreement between the three methods is excellent, of course, for [a1]. But even for [a4] where individual energies may be in error, the agreement of the distributions is very good.

quantum dot is chaotic, we know that we can use a statistical description of the eigenlevels and eigenstates of $\hat{H}_{\mathrm{TF}}=$ $\mathbf{p}^{2} / 2 m+U_{\mathrm{TF}}(\mathbf{r})$ in terms of Random Matrix Theory (RMT) and Random Plane Wave (RPW) modeling. We are therefore in the position to follow the line of reasoning in Ref. 13 to analyse the SDFT calculation. We shall do this in this section first to get some understanding of the peak spacing and spin distributions obtained, and in a later stage to address the mechanism of spin contamination.

\section{A. Universal Hamiltonian form}

To model the statistical properties obtained from the SDFT calculations, let us assume that the Thomas-Fermi density across the dot has variation small enough that we can take the parameter $r_{s}$ as a constant. We furthermore impose $n_{\mathrm{TF}}^{\alpha}(\mathbf{r})=$
$n_{\mathrm{TF}}^{\beta}(\mathbf{r})=n_{\mathrm{TF}}(\mathbf{r}) / 2\left(\int n_{\mathrm{TF}}^{\sigma}(\mathbf{r}) d r\right.$ might then be half integer for odd $N$, which is not a problem since at the Thomas-Fermi level the quantization of particle number is not playing any role) and write the second order Strutinsky correction as

$$
\Delta E^{(2)}=\frac{1}{2} \sum_{\substack{\sigma, \sigma^{\prime} \\ i, j}} f_{i \sigma} f_{j \sigma^{\prime}} M_{i, j}^{\sigma, \sigma^{\prime}}-\overline{\Delta E^{(2)}},
$$

with $f_{i \sigma}=0,1$ the occupation number of orbital $i$ with spin $\sigma$,

$$
\overline{\Delta E^{(2)}}=\frac{1}{2} \sum_{\sigma, \sigma^{\prime}} \int d \mathbf{r} d \mathbf{r}^{\prime} n_{\mathrm{TF}}^{\sigma}(\mathbf{r}) V_{\mathrm{screened}}^{\sigma, \sigma^{\prime}}\left(\mathbf{r}, \mathbf{r}^{\prime}\right) n_{\mathrm{TF}}^{\sigma^{\prime}}\left(\mathbf{r}^{\prime}\right),
$$

and

$$
M_{i, j}^{\sigma, \sigma^{\prime}}=\int d \mathbf{r} d \mathbf{r}^{\prime}\left|\phi_{i}(\mathbf{r})\right|^{2} V_{\text {screened }}^{\sigma, \sigma^{\prime}}\left(\mathbf{r}, \mathbf{r}^{\prime}\right)\left|\phi_{j}\left(\mathbf{r}^{\prime}\right)\right|^{2} .
$$

For a chaotic system, it can be shown that the fluctuations of the $M_{i, j}^{\sigma, \sigma^{\prime}}$ are small (variance of order $\sim \ln g / g^{2}$ ) and that their mean values are given by

$$
\left\langle M_{i, j}^{\sigma, \sigma^{\prime}}\right\rangle=\left[\hat{V}_{\text {screened }}^{\sigma, \sigma^{\prime}}(q=0)+\mu_{\mathrm{T}} \delta_{i j}\left\langle V_{\text {screened }}^{\sigma, \sigma^{\prime}}\right\rangle_{\mathrm{fc}}\right] / A,
$$

where $A$ is the area of the dot, $\mu_{\mathrm{T}}$ is 2 here since time reversal symmetry is preserved (but would be 1 if it were broken), and

$$
\left\langle V_{\text {screened }}^{\sigma, \sigma^{\prime}}\right\rangle_{\mathrm{fc}}=\frac{1}{2 \pi} \int_{0}^{2 \pi} d \theta \hat{V}_{\text {screened }}^{\sigma, \sigma^{\prime}}\left(\sqrt{2(1+\cos \theta)} k_{F}\right)
$$

is the average of the screened interaction over the Fermi circle (note $a_{0} k_{F}=\sqrt{2} / r_{s}$ ).

Note however that the screened interaction Eq. (26) is derived under the assumption that the oscillating part of the density integrates to zero, so that the total displaced charge providing the screening also does. Between the reference $S=0$ configuration (the TF case) and the higher $S$ ones, the total number of electrons is, of course, conserved, but not the number for each spin. It should be kept in mind, therefore, that the $q=0$ component of the density cannot be screened; this can be included simply by setting $\lambda_{s}(q=0) \equiv \lambda_{s}^{\text {bare }}(q=0)$.

The screened interaction matrix is characterized by its eigenvalues Eqs. (40) and (41). In Fig. (9 we thus plot the $r_{s}$ dependence of these quantities averaged over the Fermi circle, and compare them to their bare counterparts.

A few remarks are in order concerning this figure. First, we see that, because of the divergence of the Coulomb interaction at small $q$, screening has a drastic effect for the charge channel ${ }^{33}$ Screening is less dramatic in the spin channel, but can still be an order one effect as $r_{s}$ increases.

Furthermore, while the screening decreases the absolute strength of the interaction in the charge channel, it actually increases it in the spin channel. Indeed, since the interaction in the spin channel coming from SDFT is attractive, the charges in the bulk of the Fermi sea will, as long as this does not involve too much kinetic energy, move so as to increase the spin polarization. 


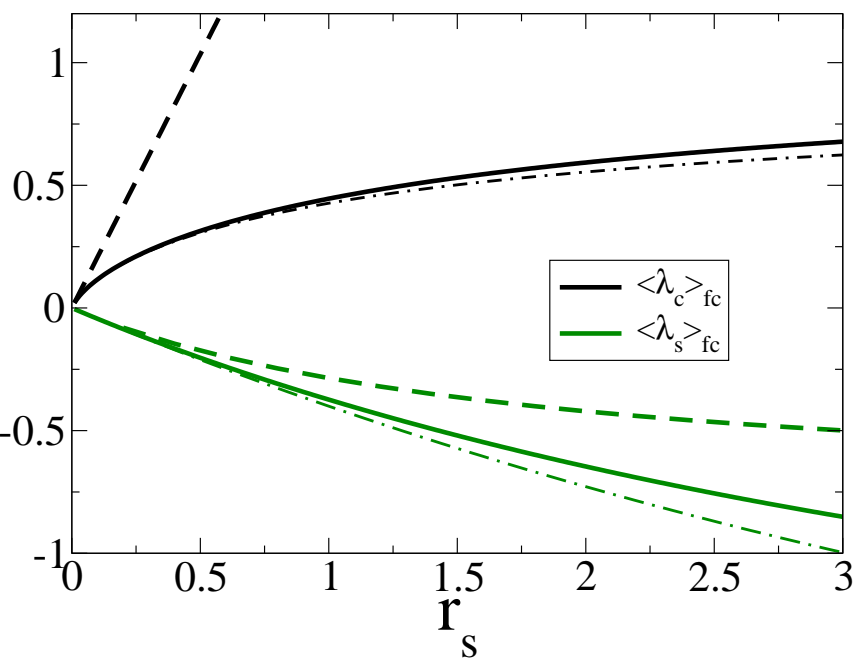

FIG. 9: (Color online) Average over the Fermi circle of the eigenvalues of the screened and bare SDFT interactions [in units of $2 / N(0)$ ] as a function of $r_{s}=\left(\pi a_{0}^{2} n\right)^{-1 / 2}$. Dark: charge channel. Lighter color (green online): spin channel. Dashed: bare interaction $\left\langle\lambda_{c, s}^{\text {bare }}\right\rangle$ (with a cutoff of the momentum at $q \simeq 1 / L$ in the charge channel). Solid: screened interaction $\left\langle\lambda_{c, s}\right\rangle$ with $\eta=0.25$. Thin dot-dashed: same but with $\eta=0$. Since the $q$ dependence of $\lambda_{s}$ is entirely due to the $\mathcal{T}_{\mathrm{TF}}^{(1)}$ correction to the Thomas-Fermi kinetic energy functional, it therefore disappears in this latter case. The interaction in the charge channel is, of course, dramatically decreased by screening; in contrast, screening increases the magnitude of the interaction in the spin chan

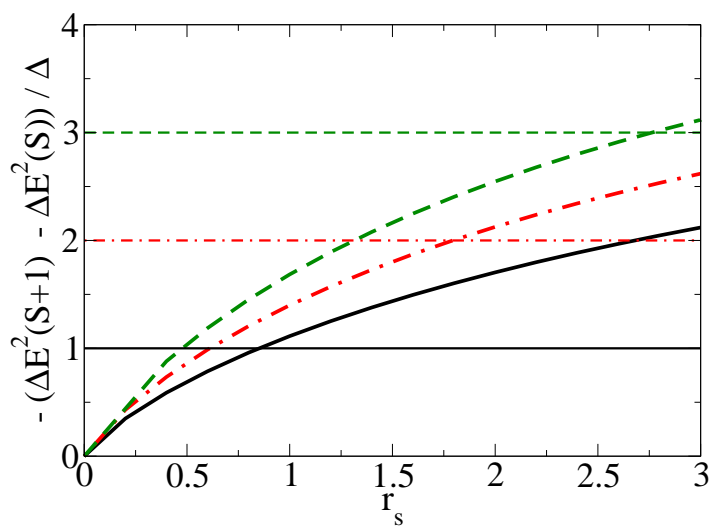

FIG. 10: (Color online) Mean value of the gain in interaction energy $-\left(\Delta E^{(2)}\left(S_{z}+1\right)-\Delta E^{(2)}\left(S_{z}\right)\right)$ (thick lines) and of the mean one-particle energy cost (thin horizontal lines) associated with flipping the spin of one particle in the quantum dot. Solid: $S_{z}=0$; Dot-Dashed $S_{z}=1 / 2$; Dashed: $S_{z}=1$

Finally, we note that for the value of the parameter $\eta$ that we use, the effect of the first $\hbar$ correction $\mathcal{T}_{\mathrm{TF}}^{(1)}$ on the screened interaction is very small in the charge channel, and only slightly larger in the spin channel.

If we neglect the fluctuations of the $M_{i, j}^{\sigma, \sigma^{\prime}}$, Eqs. 39], 45, and (48) imply that $\Delta E^{(2)}$ is just a function of the number of particles $N=N_{\alpha}+N_{\beta}$ in the dot and the $z$-component
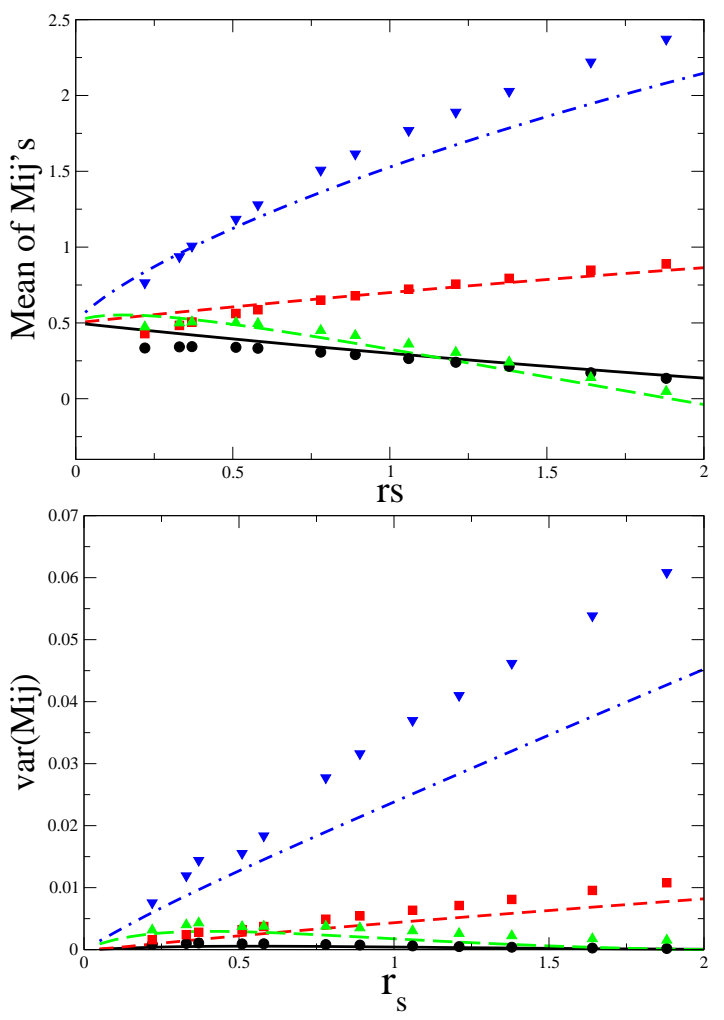

FIG. 11: (Color online) Comparison between the analytical RPW predictions [Eqs. 48 and [51] and numerical calculations of the mean and variance of $M_{i j}$ 's. The wave functions used in the numerical calculations are eigenfunctions of the effective Thomas-Fermi potential with $N=200$ in the quartic oscillator system. Lines (points) correspond to analytical (numerical) results, with the solid (circle) for $M_{i, j}^{\sigma, \sigma}$, short-dashed (square) for $M_{i, j}^{\alpha, \beta}$, long-dashed (uptriangle) for $M_{i, i}^{\sigma, \sigma}$ and dot-dashed (down-triangle) for $M_{i, i}^{\alpha, \beta}$.

$S_{z}=\left(N_{\alpha}-N_{\beta}\right) / 2$ of the groundstate the spin,

$$
\begin{array}{rl}
\Delta E^{(2)}\left(N, S_{z}\right)=\frac{1}{2} \mu_{\mathrm{T}} & N\left\langle\lambda_{c}\right\rangle_{\mathrm{fc}}+\lambda_{s}^{\text {bare }} S_{z}^{2} \\
& -\frac{\mu_{\mathrm{T}}}{2}\left(\left\langle\lambda_{c}\right\rangle_{\mathrm{fc}}-\left\langle\lambda_{s}\right\rangle_{\mathrm{fc}}\right) S_{z} .
\end{array}
$$

The main value of this expression is how it compares to the universal Hamiltonian form, ${ }^{28,29}$ and we shall come back to this point in the discussion section. Already we can see, however, that it contains almost all the information necessary to understand qualitatively the ground-state spin distributions. Indeed, looking at Fig. 10, which shows the difference $-\left(\Delta E^{(2)}\left(N, S_{z}+1\right)-\Delta E^{(2)}\left(N, S_{z}\right)\right)$ as a function of $r_{s}$ for several values of $S_{z}$, we see that for $r_{s} \simeq 0.85$, the interaction energy gain and one particle energy cost of forming a triplet are equal on average, and therefore triplets should become as probable as singlets. In the same way, spin $3 / 2$ becomes as probable as $1 / 2$ at $r_{s} \simeq 1.8$, and spin 2 as probable as 1 at $r_{s} \simeq 2.8$.

To have a more precise idea of the whether the randomplane-wave model captures the main physics, we can follow the approach of Ref. 13 and make a simulation of the peak 

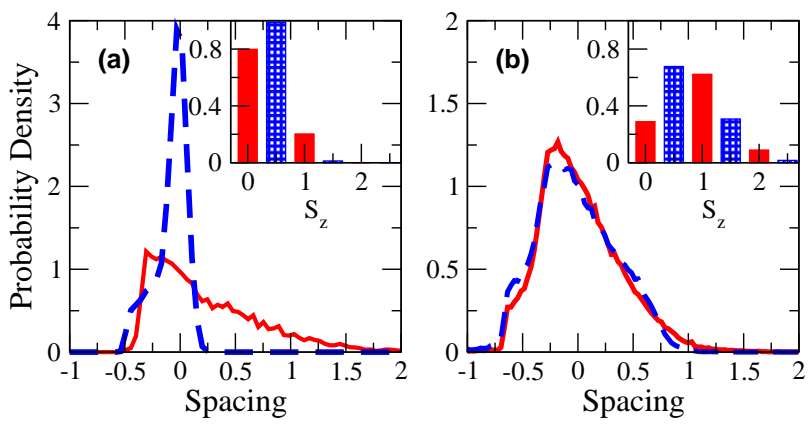

FIG. 12: (Color online) Peak spacing distributions for the RMT/RPW model corresponding to $r_{s}=0.3$ (left) and 1.3 (right). Solid : even $N$, dashed : odd $N$. Inset : corresponding spin distribution.

spacing and spin distributions. We use GOE distributed energy levels for the first-order correction Eq. 20, and take the second-order correction in the form Eq. (45) with the $M_{i, j}^{\sigma, \sigma^{\prime}}$ independent variables with mean Eq. 48 and a variance which can be computed using the method of Appendix A of Ref. 13:

$$
\begin{aligned}
& \frac{\operatorname{var}\left[M_{i, j}^{\sigma \sigma^{\prime}}\right]}{\Delta^{2}}=\frac{32}{\pi\left(k_{F} L\right)^{2}} \int_{\frac{2 \pi}{k_{F} L}}^{2-\frac{2 \pi}{k_{F} L}} \frac{d x}{x} \frac{1}{4-x^{2}} \hat{v}^{\sigma \sigma^{\prime}}(x) \\
& \quad \times\left[\hat{v}^{\sigma \sigma^{\prime}}(x)+\delta_{i j}\left(\hat{v}^{\sigma \sigma^{\prime}}(x)+\hat{v}^{\sigma \sigma^{\prime}}(0)+\hat{v}^{\sigma \sigma^{\prime}}\left(\sqrt{4-x^{2}}\right)\right)\right]
\end{aligned}
$$

where $\hat{v}^{\sigma \sigma^{\prime}}\left(q / k_{F}\right) \equiv N(0) \hat{V}_{\text {screened }}^{\sigma \sigma^{\prime}}(q) / 2$. That the randomplane-wave model correctly describe the wave function statistics is illustrated in Fig. 11] where the analytic expressions Eqs. (48) and (51) are compared, as a function of $r_{s}$, to the result from the actual eigenfunctions derived from Eq. (16). The analytic expression for the mean is expected to be quite reliable and hence the good agreement. The variance (51) is less accurate - because of the cutoff used, for instance - and so we consider the agreement in Fig. 11 quite good.

Fig. 12 displays the peak spacing and spin distribution for $r_{s}=0.3$ (corresponding to [a1]) and $r_{s}=1.3$ ([a4]) coming from a simulation in which the fluctuations of level spacing and of the $M_{i, j}^{\sigma, \sigma^{\prime}}$ are included. We see that the qualitative behavior observed in Fig 8- and in particular the lower panel is very well reproduced. Thus the RMT/RPW approach using the LSDA interaction is successful in comparison with the full SDFT calculation.

\section{B. Spin contamination}

As we saw in the previous sections, the statistical properties of the model quantum dots obtained from the full SDFT computation are, at least up to $r_{s} \simeq 1.3$, well reproduced by the various forms of the Strutinsky approximation. However, we also saw that spin contamination, when present in the SDFT calculations, was actually degrading the accuracy of the Strutinsky approximation on a case by case basis. Indeed, by construction, the Strutinsky approximation as we presented it cannot involve any spin contamination. Spin contamination is a way, in the SDFT calculations, to lower the energy of the system without changing the total $z$ component of the spin $S_{z}$ by having different wavefunctions for the $\alpha$ and $\beta$ orbitals. However, the eigenstates implicit in the Strutinsky approach are almost identical to those of $\hat{H}_{\mathrm{TF}}, \phi_{i}^{\sigma}$, and the $\phi_{i}^{\sigma}$ are nearly independent of the spin.

In this section, we shall discuss how this spin contamination mechanism could be understood within this Strutinsky scheme. Rather than trying to consider the most general situation, we will limit ourselves to the case of even number of particle $N$, and a ground state $z$ component spin equal to zero.

Let $H_{\mathrm{eff}}\left[n_{\mathrm{TF}}\right]=-\frac{\hbar^{2}}{2 m} \nabla^{2}+U_{\mathrm{TF}}^{s}(\mathbf{r})$ be the Thomas-Fermi Hamiltonian defining the orbitals $\phi_{i}^{s}$ [see Eqs. (14) and (16)]. What we have done is to construct a approximate solution of the SDFT equations Eq. (5) as $\tilde{n}^{\sigma}(\mathbf{r})=\sum_{j=1}^{N_{\sigma}} \phi_{j}^{\sigma}(\mathbf{r})$ plus some screening charge. In this respect, an important point was that the resulting potential change $\delta U^{\sigma}$ given by Eq. 31. was such that the matrix element $\left\langle\phi_{i}^{s}\left|\delta U^{\sigma}\right| \phi_{j}^{s}\right\rangle$ for $i \neq j$ was negligible, to first order in $1 / g$.

However, finding an approximate solution of the KohnSham equation implies only that one has an extremum of the spin density functional, but not necessarily an absolute minimum. As pointed out earlier, modifications of the wavefunctions change the electronic density only if they mix occupied and unoccupied orbitals. Therefore, when searching for a new extremum of the spin density functional, with some chance to actually get the true minimum, a natural choice is to mix the last occupied orbital $\phi_{M}^{\sigma}$ with the first unoccupied one $\phi_{M+1}^{\sigma}$ (for $M=N / 2=N_{\alpha}=N_{\beta}$ ).

Let us therefore look for approximations $\varphi_{i}^{\sigma}$ to the KS wavefunctions defined by $\varphi_{i}^{\sigma}=\phi_{i}^{\sigma}$ for $i<M$ and

$$
\begin{aligned}
\varphi_{M}^{\sigma} & =\cos \theta^{\sigma} \phi_{M}+\sin \theta^{\sigma} \phi_{M+1} \\
\varphi_{M+1}^{\sigma} & =-\sin \theta^{\sigma} \phi_{M}+\cos \theta^{\sigma} \phi_{M+1},
\end{aligned}
$$

with possibly a different value of the angle $\theta^{\sigma}$ for the two spins $\sigma=\alpha, \beta$. For these wavefunctions, the Thomas -Fermi Hamiltonian has a matrix element

$$
\left\langle\varphi_{M}^{\sigma}\left|\hat{H}_{\mathrm{TF}}\right| \varphi_{M+1}^{\sigma}\right\rangle=\cos \theta^{\sigma} \sin \theta^{\sigma}\left(\tilde{\epsilon}_{M+1}-\tilde{\epsilon}_{M}\right)
$$

in terms of the Thomas-Fermi energies $\tilde{\epsilon}_{M+1}$ and $\tilde{\epsilon}_{M}$.

This change in the wave functions produces a modification of the densities $\delta n^{\sigma}=\left|\varphi_{M}^{\sigma}\right|^{2}-\overline{|\varphi|^{2}}$ which, once screening is taken into account, itself generates a perturbation potential

$$
\delta U^{\sigma}(\mathbf{r})=\sum_{\sigma^{\prime}=\alpha, \beta} \int d \mathbf{r}^{\prime} V_{\text {screened }}^{\sigma, \sigma^{\prime}}\left(\mathbf{r}, \mathbf{r}^{\prime}\right) \delta n^{\sigma^{\prime}}\left(\mathbf{r}^{\prime}\right) .
$$

(The modification of the other wavefunctions, except for screening this term, does not play a role here.) The self-consistent condition for the angles $\theta^{(\alpha, \beta)}$ is that $\left\langle\varphi_{M}^{\sigma}\left|\hat{H}_{\mathrm{TF}}\right| \varphi_{M+1}^{\sigma}\right\rangle+\left\langle\varphi_{M}^{\sigma}\left|\delta U^{\sigma}(\mathbf{r})\right| \varphi_{M+1}^{\sigma}\right\rangle=0$

In order to find matrix elements of $\delta U^{\sigma}$, let us consider for a moment the case $\sigma=\alpha$. Eq. (52) implies that

$$
\varphi_{M}^{\beta}=\cos \left(\theta^{\alpha}-\theta^{\beta}\right) \varphi_{M}^{\alpha}+\sin \left(\theta^{\alpha}-\theta^{\beta}\right) \varphi_{M+1}^{\alpha}
$$


and therefore

$$
\left\langle\varphi_{M}^{\alpha}\left|\delta U^{\alpha}\right| \varphi_{M+1}^{\alpha}\right\rangle=2 \cos \left(\theta^{\alpha}-\theta^{\beta}\right) \sin \left(\theta^{\alpha}-\theta^{\beta}\right) I
$$

with

$$
\begin{aligned}
I & =\int d \mathbf{r} d \mathbf{r}^{\prime} \varphi_{M}^{\alpha}(\mathbf{r}) \varphi_{M+1}^{\alpha}(\mathbf{r}) V_{\text {screened }}^{\alpha, \beta}\left(\mathbf{r}, \mathbf{r}^{\prime}\right) \varphi_{M}^{\alpha}\left(\mathbf{r}^{\prime}\right) \varphi_{M+1}^{\alpha}\left(\mathbf{r}^{\prime}\right) \\
& \simeq\left\langle\hat{V}_{\text {screened }}^{\alpha, \beta}\right\rangle_{\mathrm{fc}} / A
\end{aligned}
$$

where the last equality applies in lowest order in $1 / g$, that is, neglecting the fluctuations.

Noting finally that the same reasoning can be applied to the $\beta$ orbitals, we find that to get an extremum for the spin density functional the angles $\theta^{\alpha}$ and $\theta^{\beta}$ should obey

$$
\begin{aligned}
& \cos \theta^{\alpha} \sin \theta^{\alpha}\left(\epsilon_{M+1}-\epsilon_{M}\right)= \\
& 2 \cos \left(\theta^{\alpha}-\theta^{\beta}\right) \sin \left(\theta^{\alpha}-\theta^{\beta}\right)\left\langle\hat{V}_{\text {screened }}^{\alpha, \beta}\right\rangle_{\mathrm{fc}} / A
\end{aligned}
$$

and the analogous equation with $\alpha$ and $\beta$ interchanged. Obvious solutions are $\left(\theta^{\alpha}=\theta^{\beta}=0\right),\left(\theta^{\alpha}=0, \theta^{\beta}=\pi / 2\right),\left(\theta^{\alpha}=\pi / 2\right.$, $\left.\theta^{\beta}=0\right)$, and $\left(\theta^{\alpha}=\theta^{\beta}=\pi / 2\right)$. The first one corresponds to the standard $S=0$ (non-spin-contaminated) solution; the other ones involve promotion of particles from the last occupied orbital to the first unoccupied one, and so are obviously of higher energy. However, other solutions may exist. Clearly they should fulfill

$$
\cos \theta^{\alpha} \sin \theta^{\alpha}=\cos \theta^{\beta} \sin \theta^{\beta}
$$

that is, up to an irrelevant multiple of $\pi$ phase

$$
\theta^{\beta}=-\theta^{\alpha},
$$

and therefore

$$
\cos 2 \theta^{\alpha}=-\frac{\epsilon_{M+1}-\epsilon_{M}}{4\left\langle\hat{V}_{\text {screened }}^{\alpha, \beta}\right\rangle_{\mathrm{fc}} / A} .
$$

It can be checked that whenever the condition Eq. 61) can be fulfilled [ie. when $\epsilon_{M+1}-\epsilon_{M} \leq 4\left\langle\hat{V}_{\text {screened }}^{\alpha, \beta}\right\rangle_{\text {fc }}$ ], the corresponding extremum has an energy smaller by a quantity $\sin ^{2}\left(2 \theta^{\alpha}\right)\left[\left\langle\lambda_{s}\right\rangle_{\mathrm{fc}}-\left\langle\lambda_{c}\right\rangle_{\mathrm{fc}}\right] / A$ with respect to the non contaminated configuration. In this situation, the spin contaminated $S_{z}=0$ state will be favored (but its energy still needs to be compared to the lowest energy state with $S_{z}=1$ ).

\section{DISCUSSION}

To summarize our findings, we have seen that up to $r_{s}$ values of order one (in practice, 1.3 here), the Strutinsky approximation yields a ground state total energy with fluctuating part reliable up to typically five percent of the mean level spacing. Furthermore, these errors can be related to the occurrence of spin contamination in the SDFT calculation, which cannot be reproduced by the Strutinsky scheme (in its simplest form). Indeed, as discussed in the last section, this latter gives, by construction, an approximation to an extremum of the Kohn-Sham functional for which the $\alpha$ and $\beta$ orbitals are

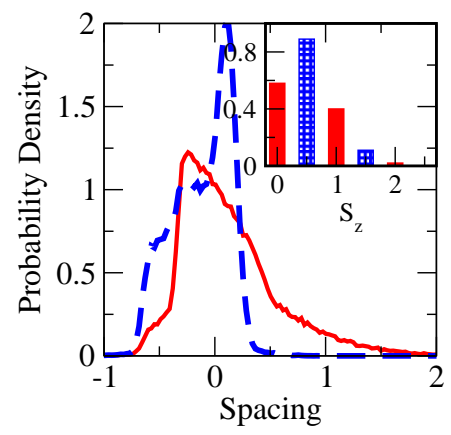

FIG. 13: (Color online) Same as Fig. 12 b), but with a residual interaction modeled by a perturbative calculation in the interaction potential $V_{\mathrm{RPA}}\left(\mathbf{r}-\mathbf{r}^{\prime}\right)$ whose parameters are set by Eqs. 64. and 65.

nearly identical, but not necessarily an approximation to the true minimum.

Nevertheless, the qualitative properties of the peak spacing and spin distribution are correctly reproduced by the Strutinsky approximation (at least up to $r_{s}$ of order one, for which spin contamination does not appear to change drastically the distributions). For a chaotic confining potential this makes it possible to use the modeling in terms of Random Matrix Theory (for the energy levels) and Random Plane Waves (for the eigenstates) introduced in Ref. 13. Within this RMT/RPW modeling, and in the limit of large dots for which fluctuations of the residual interaction term are small, the main features of the peak spacing and spin distributions can be understood as arising from the interplay between one particle level fluctuations and the spin dependence of the mean residual interaction term [see Eq. [50)]

$$
\begin{aligned}
\overline{\Delta E_{\mathrm{SDFT}}^{(2)}}\left(N, S_{z}\right)= & \lambda_{s}^{\mathrm{bare}} S_{z}^{2}-\frac{\mu_{\mathrm{T}}}{2}\left(\left\langle\lambda_{c}\right\rangle_{\mathrm{fc}}-\left\langle\lambda_{s}\right\rangle_{\mathrm{fc}}\right) S_{z} \\
& +(\text { term depending on } N \text { only }) .(62)
\end{aligned}
$$

It is useful to compare this expression, as well as the corresponding distributions based on RMT/RPW modeling such as those in Fig. 12 (which actually take into account the fluctuations of the residual interaction term), to what is obtained following the more traditional route to the analysis of peak spacing and spin distributions for chaotic quantum dots 12.13 .15 In these earlier approaches, the ground state energy of the quantum dot would, in a way very similar way to Eq. (19), be described as the sum of a large non-fluctuating classical-like term, a one-particle energy contribution computed for some effective confining potential, and finally a residual interaction term $\Delta E_{\mathrm{RPA}}^{(2)}$. This latter would, however, be understood as originating from a weak interaction $V_{\mathrm{RPA}}\left(\mathbf{r}-\mathbf{r}^{\prime}\right)$ which in the Random Phase Approximation can be shown to be just the RPA screened potential, ${ }^{12,15}$ but should be understood more properly as the residual interaction between quasi-particles in Landau Fermi liquid spirit. We shall thus refer to this latter description as the "RPA" approach, although this is slightly inappropriate.

While the Strutinsky approximation to SDFT gives a residual interaction which can be understood as a first-order perturbation (without exchange) in terms of the spin dependent 


\begin{tabular}{|c|ccccc|}
\hline model & $S=0$ & $S=\frac{1}{2}$ & $S=1$ & $S=\frac{3}{2}$ & $S=2$ \\
\hline SDFT & $0.42 \pm 0.03$ & $0.76 \pm 0.03$ & $0.54 \pm 0.03$ & $0.23 \pm 0.03$ & $0.03 \pm 0.01$ \\
ST $^{*}$ & $0.34 \pm 0.03$ & $0.74 \pm 0.03$ & $0.61 \pm 0.03$ & $0.25 \pm 0.03$ & $0.04 \pm 0.01$ \\
ST $^{*} / \mathrm{RPW}$ & 0.28 & 0.68 & 0.62 & 0.30 & 0.09 \\
RPA/RPW (unscreened Cooper) & 0.48 & 0.84 & 0.49 & 0.16 & 0.03 \\
RPA/RPW (screened Cooper ) & 0.58 & 0.89 & 0.40 & 0.11 & 0.02 \\
\hline
\end{tabular}

TABLE I: Ground state spin probability for various model introduced in this paper. The two first lines correspond respectively to the SDFT calculation and [second form of] Strutinsky approximation for the quartic oscillator systems introduced in section IV The statistics are built from the ground state spin of dots containing 100 to 200 electrons, for a few confining potential corresponding to an interaction parameter $r_{s} \simeq 1.3$. The three last lines are the results of RMT/RPW modeling for 150 electron and the same value of the interaction parameter $r_{s}$, and respectively: the interaction derived from SDFT (third line); the "RPA"-like interaction using a screened Cooper channel, i.e. such that Eqs. 64 and 65 apply (fourth line); and the "RPA"-like interaction using a unscreened Cooper channel, i.e. such that Eqs. 64 applies but $\zeta=J_{S}$ (fifth line).

potential Eq. 26, in contrast one has in the "RPA" approach a residual interaction arising from the perturbative corrections in some $V_{\mathrm{RPA}}\left(\mathbf{r}, \mathbf{r}^{\prime}\right)$ (including both direct and exchange) as well as possibly higher-order terms which turn out to be important for time-reversal invariant systems (the Cooper series). Under this assumption and following exactly the same analysis as the one leading to Eq. 62, one gets for the mean residual interaction term in the RPA approach

$$
\begin{aligned}
\overline{\Delta E_{\mathrm{RPA}}^{(2)}}\left(N, S_{z}\right)= & J_{S} S_{z}\left(S_{z}+1\right)+\zeta\left(\mu_{\mathrm{T}}-1\right) S_{z} \\
& +(\text { term depending on } N \text { only })
\end{aligned}
$$

(again $\mu_{\mathrm{T}}=2$ but would be 1 if time-reversal invariance were broken).

In Eq. 63, the parameter $J_{S}$ is equal to $-\langle\hat{V}(q)\rangle_{\mathrm{fc}}$, where the Fermi circle average is defined by Eq. (49) and $\hat{V}_{\mathrm{RPA}}(\mathbf{q})$ is the Fourier transform of $V_{\mathrm{RPA}}\left(\mathbf{r}-\mathbf{r}^{\prime}\right)$. More properly however, one should understand $J_{S}$ as being related to Fermi liquid parameter $f_{0}^{(a)}$ through

$$
J_{S} / \Delta=f_{0}^{(a)} .
$$

In first-order perturbation theory, $\zeta$ would be equal to $J_{S}$, but screening associated with higher oder terms in the Cooper channel somewhat reduces this value. An analysis following the lines of Ref. 30 suggests that

$$
\zeta=\frac{J_{s}}{1+\frac{\left|J_{S}\right|}{\Delta} \log \left(k_{F} L\right)},
$$

with $k_{F}$ the Fermi momentum and $L$ the typical size of the system. We shall assume this in the following discussion, bearing, in mind that this is true only "up to logarithmic accuracy".

The remarkable point here is that $\lambda_{s}^{\text {bare }}\left(r_{s}\right) / \Delta$ is actually the same thing as $f_{0}^{(a)}\left(r_{s}\right)$, in the sense that the Landau Fermi liquid parameter $f_{0}^{(a)}\left(r_{s}\right)$ can be interpreted as the second derivative with respect to the polarization, at fixed total density, of $\epsilon_{\mathrm{xc}}$ the exchange correlation energy per particle of the uniform electron gas. This implies that the term quadratic in $S_{z}$ of Eqs. 62) and (63) actually do correspond.
As a consequence if we compare Fig. 112 b), which is obtained from a RMT/RPW simulation with the interaction corresponding to the spin density functional, with Fig. 13, obtained in the same way but with an "RPA"-like interaction, 34 the differences in spin polarization and in odd/even asymmetry for the peak spacing distribution can be almost entirely associated with the different linear $S_{z}$ terms in Eqs. (62) and 63.

To get some further insight into the difference between the two approaches, let us consider Table \which shows the spin distributions for an interaction parameter $r_{s} \simeq 1.3$ and various approximations discussed in this paper. Comparing different pairs of lines gives a sense of the importance of the various issues. For instance, comparing the second line with the third gives an idea of how accurate the RMT/RPW model is for the statistical properties of the real energy levels and eigenfunctions of the quartic oscillator system, since both lines are based on the same spin dependent interaction Eq. (26). The first line compared to the second, on the other hand, is a measure of how well the Strutinsky scheme approximates the full SDFT calculation. Presumably, most of the difference between these two lines can be associated with the presence of spin contamination in SDFT - it is in some sense a measure of the effectiveness of spin contamination in lowering the total spin of the system. Going further down the table, the difference between the third and the two last lines is a measure of the impact of different linear terms in $S_{z}$ in Eqs. (62) and 63, without screening the Cooper channel for the fourth line and with a screened Cooper channel [according to Eq. 65] ] for the last line of the table.

From Table 【 it appears that within the accuracy of the RMT/RPW modeling, which seems to be around 5\%, the SDFT result is compatible with an RPA-like approach if the Cooper channel is not screened. In other words, the fact that $\left(\left\langle\lambda_{s}\right\rangle_{\mathrm{fc}}-\left\langle\lambda_{c}\right\rangle_{\mathrm{fc}}\right) / 2$ is more negative that $J_{s}=\lambda_{s}^{\text {bare }}$ - producing higher spins - is compensated by the effect of spin contamination - which favors lower spins. As seen in Ref.31, this compensation between the two effects seems to exist also for higher values of $r_{s}$. On the other hand, spin contamination is not a sufficiently strong effect to compensate for the absence of screening of the Cooper channel.

It remains to decide which of the two approaches is the 
more correct. This, in the end, can only be addressed by comparison with exact calculations for quantum dots (e.g. quantum Monte Carlo). One argument that may be considered is that in the presence of a time-reversal breaking term (i.e. $\mu_{\mathrm{T}}=1$ ), general symmetry considerations impose that the mean value of the residual interaction term is a function of $S(S+1)$, but not independently of $S^{2}$ and $S$. Expressions (62) clearly do not fulfill this constraint, while Eq. 63. does. Since, however, spin contamination seems to compensate for this difference it might just be that for time-reversal non-symmetric systems, SDFT and RPA basically agree. On the other hand, the screening of the Cooper channel does not seem to be reproduced by the SDFT calculations, and this might be the cause of the higher spin found in this approach.

\section{Acknowledgments}

We thank G. Usaj for several valuable conversations and M. Brack for helpful comments regarding $\hbar$ corrections. This work was supported in part by the NSF (DMR-0103003).
${ }^{1}$ L. P. Kouwenhoven, C. M. Marcus, P. L. McEuen, S. Tarucha, R. M. Wetervelt, and N. S. Wingreen, in Mesoscopic electron transport, edited by L. L. Sohn, G. Schön, and L. P. Kouwenhoven (Kluwer, Dordrecht, 1997), pp. 105-214.

2 Y. Alhassid, Rev. Mod. Phys. 72, 895 (2000), and references therein.

3 D. Weinmann, W. Hsler, and B. Kramer Phys. Rev. Lett. 74, 984987 (1995).

${ }^{4}$ E. R. Mucciolo, C. Chamon, and C. M. Marcus Phys. Rev. Lett. 89, 146802 (2002).

5 D. Loss and D. P. DiVincenzo, Phys. Rev. A 57, 120 (1998).

${ }^{6}$ R. G. Parr and W. Yang, Density-Functional Theory of Atoms and Molecules (Oxford University Press, New York, 1989).

7 R. O. Jones and O. Gunnarsson, Rev. Mod. Phys. 61, 689, (1989).

${ }^{8}$ S. M. Reimann and M. Manninen, Rev. Mod. Phys. 74, 1283 (2002), and references therein.

9 H. Jiang, H. U. Baranger and W. Yang, Phys. Rev. Lett. 90, 026806 (2003)

${ }^{10}$ H. Jiang, H. U. Baranger and W. Yang, Phys. Rev. B 68, 165337 (2003)

11 H. Jiang, D. Ullmo, W. Yang, and H. U. Baranger, preprint (cond-mat/0401043).

12 Y. M. Blanter, A. D. Mirlin, and B. A. Muzykantskii, Phys. Rev. Lett. 78, 2449 (1997).

13 D. Ullmo and H. U. Baranger, Phys. Rev. B 64, 245324 (2001).

14 G. Usaj and H. U. Baranger, Phys. Rev. B 63, 184418 (2001); ibid. 66, 155333 (2002).

15 I. L. Aleiner, P. W. Brouwer, and L. I. Glazman, Phys. Rep. 358, 309 (2002).

16 D. Ullmo, T. Nagano, S. Tomsovic, and H. U. Baranger, Phys. Rev. B 63, 125339 (2001).

17 V. M. Strutinsky, Nucl. Phys. A122, 1 (1968).

${ }^{18}$ M. Brack, J. Damgaard, A. S. Jensen, H. C. Pauli, V. M. Strutinsky, and C. Y. Wong, Rev. Mod. Phys. 44, 320 (1972).

19 M. Brack and R. K. Bhaduri, Semiclassical Physics (AddisonWesley, Reading MA, 1997).

${ }^{20}$ C. Yannouleas and U. Landman Phys. Rev. B 48, 8376 (1993).

21 J. Harris, Phys. Rev. B 31, 1770 (1985).

22 This second order Strutinsky approximation has to be distinguished from second order approximations made on the the density functional itself, such as in : M. Elstner, D. Porezag, G. Jungnickel, J. Elsner, M. Haugk, T. Frauenheim, S. Suhai, and G. Seifert, Phys. Rev. B58 7260-7268 (1998).

23 B. Tanatar and D. M. Ceperley, Phys. Rev. B 39, 5005 (1989).

${ }^{24}$ C. Attaccalite, S. Moroni, P. Gori-Giorgi and G. B. Bachelet, Phys. Rev. Lett. 88, 256601 (2002).

${ }^{25}$ H. Saarikoski, E. Räsänen, S. Siljamäki, A. Harju, M. J. Puska, R. M. Nieminen, Phys. Rev. B 67, 205327 (2003).

${ }^{26} \mathrm{H}$. Jiang and W. Yang, submitted to J. Chem. Phys (condmat/0401337).

27 J. Wang, A. D. Becke and V. H. Smith, Jr., J. Chem. Phys. 102 3477 (1995).

${ }^{28}$ I. L. Kurland, I. L. Aleiner, and B. L. Altshuler, Phys. Rev. B 62, 14886-14897 (2000).

${ }^{29}$ I. L. Aleiner, P. W. Brouwer, and L. I. Glazman, Phys. Rep. 358, 309 (2002)

${ }^{30}$ D. Ullmo, H. U. Baranger, K. Richter, F. von Oppen and R. A. Jalabert, Phys. Rev. Lett. 80895 (1998).

${ }^{31}$ H. Jiang, D. Ullmo, W. Yang, and H. U. Baranger, submitted to Phys. Rev. B (cond-mat/0401043).

${ }^{32}$ For the higher density case [a1], it was actually necessary to place the gate quite close to the electron gas to see any effect on the density, even on the classical scale. The image charge associated with the gate is at a distance $2 z_{0}$ which is, however, still larger than the screening length within the electron gas.

33 Actually $\left\langle\lambda_{c}^{\text {bare }}\right\rangle_{\mathrm{fc}}$ would be divergent if we did not use a cutoff on $q$ at the inverse of the system size.

${ }^{34}$ For this simulation, the fluctuation of the residual interaction term also depends on the precise way the Cooper channel contribution is screened. Fig. 12 corresponds to an evaluation of the variance of these fluctuations for which we have assumed no screening, and is therefore rather an upper bound. The fluctuations being in any case rather small, their magnitude does not change the peak spacing distribution drastically (their main effect is to broaden the sharp peak for odd spacings), and a more detailed treatment of the effect of screening of the Cooper channel on the fluctuations of the residual interaction term should not change significantly the picture. 